\title{
Optimal Approximate Sampling from Discrete Probability Distributions
}

\author{
FERAS A. SAAD, Massachusetts Institute of Technology, USA \\ CAMERON E. FREER, Massachusetts Institute of Technology, USA \\ MARTIN C. RINARD, Massachusetts Institute of Technology, USA \\ VIKASH K. MANSINGHKA, Massachusetts Institute of Technology, USA
}

This paper addresses a fundamental problem in random variate generation: given access to a random source that emits a stream of independent fair bits, what is the most accurate and entropy-efficient algorithm for sampling from a discrete probability distribution $\left(p_{1}, \ldots, p_{n}\right)$, where the probabilities of the output distribution $\left(\hat{p}_{1}, \ldots, \hat{p}_{n}\right)$ of the sampling algorithm must be specified using at most $k$ bits of precision? We present a theoretical framework for formulating this problem and provide new techniques for finding sampling algorithms that are optimal both statistically (in the sense of sampling accuracy) and information-theoretically (in the sense of entropy consumption). We leverage these results to build a system that, for a broad family of measures of statistical accuracy, delivers a sampling algorithm whose expected entropy usage is minimal among those that induce the same distribution (i.e., is "entropy-optimal") and whose output distribution $\left(\hat{p}_{1}, \ldots, \hat{p}_{n}\right)$ is a closest approximation to the target distribution $\left(p_{1}, \ldots, p_{n}\right)$ among all entropy-optimal sampling algorithms that operate within the specified $k$-bit precision. This optimal approximate sampler is also a closer approximation than any (possibly entropy-suboptimal) sampler that consumes a bounded amount of entropy with the specified precision, a class which includes floating-point implementations of inversion sampling and related methods found in many software libraries. We evaluate the accuracy, entropy consumption, precision requirements, and wall-clock runtime of our optimal approximate sampling algorithms on a broad set of distributions, demonstrating the ways that they are superior to existing approximate samplers and establishing that they often consume significantly fewer resources than are needed by exact samplers.

CCS Concepts: • Theory of computation $\rightarrow$ Probabilistic computation; Numeric approximation algorithms; • Mathematics of computing $\rightarrow$ Probability and statistics; Random number generation; Mathematical software performance; Combinatorial optimization; Discretization.

Additional Key Words and Phrases: random variate generation, discrete random variables

\section{ACM Reference Format:}

Feras A. Saad, Cameron E. Freer, Martin C. Rinard, and Vikash K. Mansinghka. 2020. Optimal Approximate Sampling from Discrete Probability Distributions. Proc. ACM Program. Lang. 4, POPL, Article 36 (January 2020), 31 pages. https://doi.org/10.1145/3371104

Authors' addresses: Feras A. Saad, Department of Electrical Engineering \& Computer Science, Massachusetts Institute of Technology, Cambridge, MA, 02139, USA, fsaad@mit.edu; Cameron E. Freer, Department of Brain \& Cognitive Sciences, Massachusetts Institute of Technology, Cambridge, MA, 02139, USA, freer@mit.edu; Martin C. Rinard, Department of Electrical Engineering \& Computer Science, Massachusetts Institute of Technology, Cambridge, MA, 02139, USA, rinard@ csail.mit.edu; Vikash K. Mansinghka, Department of Brain \& Cognitive Sciences, Massachusetts Institute of Technology, Cambridge, MA, 02139, USA, vkm@mit.edu.

This work is licensed under a Creative Commons Attribution-NonCommercial-ShareAlike 4.0 International License. (C) 2020 Copyright held by the owner/author(s).

2475-1421/2020/1-ART36

https://doi.org/10.1145/3371104

Proc. ACM Program. Lang., Vol. 4, No. POPL, Article 36. Publication date: January 2020. 


\section{INTRODUCTION}

Sampling from discrete probability distributions is a fundamental activity in fields such as statistics [Devroye 1986], operations research [Harling 1958], statistical physics [Binder 1986], financial engineering [Glasserman 2003], and general scientific computing [Liu 2001]. Recognizing the importance of sampling from discrete probability distributions, widely-used language platforms [Lea 1992; MathWorks 1993; R Core Team 2014] typically implement algorithms for sampling from discrete distributions. As Monte Carlo methods move towards sampling billions of random variates per second [Djuric 2019], there is an increasing need for sampling algorithms that are both efficient (in terms of the number of random bits they consume to generate a sample) and accurate (in terms of the statistical sampling error of the generated random variates with respect to the intended probability distribution). For example, in fields such as lattice-based cryptography and probabilistic hardware [de Schryver et al. 2012; Roy et al. 2013; Dwarakanath and Galbraith 2014; Folláth 2014; Mansinghka and Jonas 2014; Du and Bai 2015], the number of random bits consumed per sample, the size of the registers that store and manipulate the probability values, and the sampling error due to approximate representations of numbers are all fundamental design considerations.

We evaluate sampling algorithms for discrete probability distributions according to three criteria: (1) the entropy consumption of the sampling algorithm, as measured by the average number of random bits consumed from the source to produce a single sample (Definition 2.5); (2) the error of the sampling algorithm, which measures how closely the sampled probability distribution matches the specified distribution, using one of a family of statistical divergences (Definition 4.2); and (3) the precision required to implement the sampler, as measured by the minimum number of binary digits needed to represent each probability in the implemented distribution (Definition 2.13).

Let $\left(M_{1}, \ldots, M_{n}\right)$ be a list of $n$ positive integers which sum to $Z$ and write $\mathbf{p}:=\left(p_{1}, \ldots, p_{n}\right)$ for the discrete probability distribution over the set $[n]:=\{1, \ldots, n\}$ defined by $p_{i}:=M_{i} / Z(i=1, \ldots, n)$. We distinguish between two types of algorithms for sampling from p: (i) exact samplers, where the probability of returning $i$ is precisely equal to $p_{i}$ (i.e., zero sampling error); and (ii) approximate samplers, where the probability of returning $i$ is $\hat{p}_{i} \approx p_{i}$ (i.e., non-zero sampling error). In exact sampling, the numerical precision needed to represent the output probabilities of the sampler varies with the values $p_{i}$ of the target distribution; we say these methods need arbitrary precision. In approximate sampling, on the other hand, the numerical precision needed to represent the output probabilities $\hat{p}_{i}$ of the sampler is fixed independently of the $p_{i}$ (by constraints such as the register width of a hardware circuit or arithmetic system implemented in software); we say these methods need limited precision. We next discuss the tradeoffs between entropy consumption, sampling error, and numerical precision made by exact and approximate samplers.

\subsection{Existing Methods for Exact and Approximate Sampling}

Inversion sampling is a universal method for obtaining a random sample from any probability distribution [Devroye 1986, Theorem 2.1]. The inversion method is based on the identity that if $U$ is a uniformly distributed real number on the unit interval $[0,1]$, then

$$
\operatorname{Pr}\left[\sum_{i=1}^{j-1} p_{i} \leq U<\sum_{i=1}^{j} p_{i}\right]=p_{j} \quad(j=1, \ldots, n) .
$$

Knuth and Yao [1976] present a seminal theoretical framework for constructing an exact sampler for any discrete probability distribution. The sampler consumes, in expectation, the least amount of random bits per sample among the class of all exact sampling algorithms (Theorem 2.9). The Knuth and Yao sampler is an implementation of the inversion method which compares (lazily sampled) bits in the binary expansion of $U$ to the bits in the binary expansion of the $p_{i}$. Despite its minimal entropy consumption and zero sampling error, the method requires arbitrary precision and the 


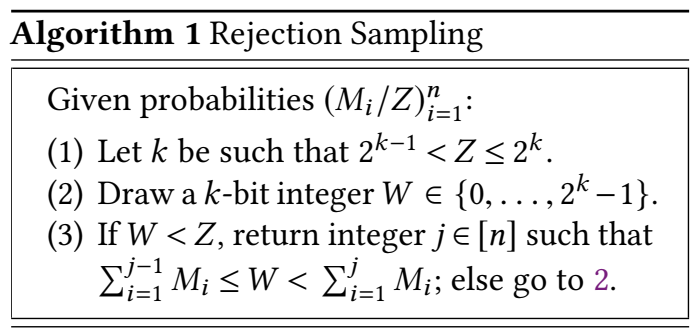

\begin{tabular}{l}
\hline Algorithm 2 Inversion Sampling \\
\hline \hline Given probabilities $\left(M_{i} / Z\right)_{i=1}^{n}$, precision $k:$ \\
(1) Draw a $k$-bit integer $W \in\left\{0, \ldots, 2^{k}-1\right\}$. \\
(2) Let $U^{\prime}:=W / 2^{k}$. \\
(3) Return smallest integer $j \in[n]$ such that \\
$U^{\prime}<\sum_{i=1}^{j} M_{i} / Z$. \\
\hline \hline
\end{tabular}

computational resources needed to implement the sampler are often exponentially larger than the number of bits needed to encode the probabilities (Theorem 3.5), even for typical distributions (Table 4). In addition to potentially requiring more resources than are available even on modern machines, the framework is presented from a theoretical perspective without readily-programmable implementations of the sampler, which has further limited its general application. ${ }^{1}$

The rejection method [Devroye 1986], shown in Algorithm 1, is another technique for exact sampling where, unlike the Knuth and Yao method, the required precision is polynomial in the number of bits needed to encode $\mathbf{p}$. Rejection sampling is exact, readily-programmable, and typically requires reasonable computational resources. However, it is highly entropy-inefficient and can consume exponentially more random bits than is necessary to generate a sample (Example 5.1).

We now discuss approximate sampling methods which use a limited amount of numerical precision that is specified independently of the target distribution p. Several widely-used software systems such as the MATLAB Statistics Toolbox [MathWorks 1993] and GNU C++ standard library [Lea 1992] implement the inversion method based directly on Eq. (1), where a floating-point number $U^{\prime}$ is used to approximate the ideal real random variable $U$, as shown in Algorithm 2. These implementations have two fundamental deficiencies: first, the algorithm draws a fixed number of random bits (typically equal to the 32-bit or 64-bit word size of the machine) per sample to determine $U^{\prime}$, which may result in high approximation error (Section 2.4), is suboptimal in its use of entropy, and often incurs non-negligible computational overhead in practice; second, floating-point approximations for computing and comparing $U^{\prime}$ to running sums of $p_{i}$ produce significantly suboptimal sampling errors (Figure 3) and the theoretical properties are challenging to characterize [von Neumann 1951; Devroye 1982; Monahan 1985]. In particular, many of these approximate methods, unlike the method presented in this paper, are not straightforwardly described as producing samples from a distribution that is close to the target distribution with respect to a specified measure of statistical error and provide no optimality guarantees.

The interval method [Han and Hoshi 1997] is an implementation of the inversion method which, unlike the previous methods, lazily obtains a sequence $U_{i}$ of fair coin flips from the set $\{0,1\}$ and recursively partitions the unit interval until the outcome $j \in[n]$ can be determined. Han and Hoshi [1997] present an exact sampling algorithm (using arbitrary precision) and Uyematsu and Li [2003] present an approximate sampling algorithm (using limited precision). Although entropy consumed by the interval method is close to the optimal limits of Knuth and Yao [1976], the exact sampler uses several floating-point computations and has an expensive search loop during sampling [Devroye and Gravel 2015, Algorithm 1]. The limited-precision sampler is more entropy-efficient than the limited-precision inversion sampler (Table 2) but often incurs a higher error (Figure 3).

\footnotetext{
${ }^{1}$ In reference to the memory requirements and programmability of the Knuth and Yao [1976] method, the authors note "most of the algorithms which achieve these optimum bounds are very complex, requiring a tremendous amount of space". Lumbroso [2013] also discusses these issues.
} 


\subsection{Optimal Approximate Sampling}

This paper presents a novel class of algorithms for optimal approximate sampling from discrete probability distributions. Given a target distribution $\mathbf{p}:=\left(p_{1}, \ldots, p_{n}\right)$, any measure of statistical error in the family of (1-1 transformations of) $f$-divergences (Definition 4.2 ), and a number $k$ specifying the allowed numerical precision, our system returns a sampler for $\mathbf{p}$ that is optimal in a very strong sense: it produces random variates with the minimal sampling error possible given the specified precision, among the class of all entropy-optimal samplers of this precision (Theorems 3.4 and 4.7). Moreover these samplers comprise, to the best of our knowledge, the first algorithms that, for any target distribution, measure of statistical accuracy, and specification of bit precision, provide rigorous guarantees on the entropy-optimality and the minimality of the sampling error.

The key idea is to first find a distribution $\hat{\mathbf{p}}:=\left(\hat{p}_{1}, \ldots, \hat{p}_{n}\right)$ whose approximation error of $\mathbf{p}$ is minimal among the class of all distributions that can be sampled by any $k$-bit entropy-optimal sampler (Section 4). The second step is to explicitly construct an entropy-optimal sampler for the distribution $\hat{\mathbf{p}}$ (Section 5). In comparison with previous limited-precision samplers, our samplers are more entropy-efficient and more accurate than any sampler that always consumes at most $k$ random bits (Proposition 2.16), which includes any algorithm that uses a finite number of approximately uniform floating-point numbers (e.g., limited-precision inversion sampling and interval sampling) The time, space, and entropy resources required by our samplers can be significantly less than those required by the exact Knuth and Yao and rejection methods (Section 6.3), with an approximation error that decreases exponentially quickly with the amount of precision (Theorem 4.17).

The sampling algorithms delivered by our system are algorithmically efficient: they use integer arithmetic, admit straightforward implementations in software and probabilistic hardware systems, run in constant time with respect to the length $n$ of the target distribution and linearly in the entropy of the sampler, and can generate billions of random variates per second. In addition, we present scalable algorithms where, for a precision specification of $k$ bits, the runtime of finding the $n$ optimal approximate probabilities $\hat{\mathbf{p}}$ is order $n \log n$, and of building the corresponding sampler is order $n k$. Prototype implementations of the system in $\mathrm{C}$ and Python are available in the online artifact and at https://github.com/probcomp/optimal-approximate-sampling.

\subsection{Contributions}

The main contributions of this paper are:

Formulation of optimal approximate sampling algorithms for discrete distributions. This precise formulation allow us to rigorously study the notion of entropy consumption, statistical sampling error, and numerical precision. These three functional metrics are used to assess the entropy-efficiency, accuracy, and memory requirements of a sampling algorithm.

Theoretical results for the class of entropy-optimal sampling algorithms. For a specified precision, we characterize the set of output probability distributions achievable by any entropyoptimal sampler that operates within the given precision specification. We leverage these results to constrain the space of probability distributions for approximating a given target distribution to contain only those that correspond to limited-precision entropy-optimal samplers.

Algorithms for finding optimal approximations to discrete distributions. We present a new optimization algorithm that, given a target distribution $\mathbf{p}$, a measure of statistical divergence, and a precision specification, efficiently searches the combinatorially large space of entropy-optimal samplers of the given precision, to find a optimal approximation sampler that most accurately approximates the target distribution $\mathbf{p}$. We prove the correctness of the algorithm and analyze its runtime in terms of the size of the target distribution and precision specification. 
Algorithms for constructing entropy-optimal sampling algorithms. We present detailed algorithms for sampling from any closest-approximation probability distribution $\hat{\mathbf{p}}$ in a way that is entropy-optimal, using the guarantees provided by the main theorems of Knuth and Yao [1976] Our prototype implementation can generate billions of random variates per second and executes between 1.5x (for low-dimensional distributions) and 195x (for high-dimensional distributions) faster than the limited-precision linear inversion sampler provided as part of the GNU C++ standard library [Lea 1992].

Comparisons to baseline limited-precision sampling algorithms. For several common probability distributions, we empirically demonstrate that the proposed sampling algorithms consume less entropy and are up to $1000 \mathrm{x}-10000 \mathrm{x}$ more accurate than the limited-precision inversion sampler from the GNU C++ standard library [Lea 1992] and interval algorithm [Uyematsu and Li 2003]. We also show that (i) our sampler scales more efficiently as the size of the target distribution grows; and (ii) using the information-theoretically minimal amount of bits per sample leads to up to $10 \mathrm{x}$ less wall-clock time spent calling the underlying pseudorandom number generator.

Comparisons to baseline exact sampling algorithms. We present a detailed study of the exact Knuth and Yao method, the rejection method, and the proposed method for a canonical discrete probability distribution. We demonstrate that our samplers can use 150x less random bits per sample than rejection sampling and many orders of magnitude less precision than exact Knuth and Yao sampling, and can (unlike exact sampling algorithms) trade off greater numerical precision in exchange for exponentially smaller sampling accuracy, all while remaining entropy-optimal.

The remainder of this paper is structured as follows: Section 2 describes the random bit model of computation for sampling algorithms and provides formal definitions used throughout the paper. Section 3 presents theoretical results on the class of entropy-optimal samplers which are leveraged in future sections. Section 4 presents an efficient algorithm for finding a closest-approximation distribution to any given target distribution. Section 5 presents algorithms for constructing entropyoptimal samplers. Section 6 investigates the properties of the optimal samplers and compares them to multiple existing sampling methods in terms of accuracy, precision, entropy, and runtime.

\section{COMPUTATIONAL MODELS OF SAMPLING ALGORITHMS}

In the algebraic model of computation over the real numbers (also known as the real RAM model [Blum et al. 1998]), a sampling algorithm has access to an ideal register machine that can (i) sample a real random variable $U$ uniformly distributed on the unit interval $[0,1]$ using a primitive called uniform(), which forms the basic unit of randomness; and (ii) store and perform algebraic operations on infinitely-precise real numbers in unit time [Devroye 1986, Assumptions 1, 2, and 3]. The algebraic model is useful for proving the correctness of exact mathematical transformations applied to a uniform random variate $U$ and for analyzing the algorithmic runtime and storage costs of preprocessing and sampling, assuming access to infinite amounts of entropy or precision [Walker 1977; Vose 1991; Smith 2002; Bringmann and Panagiotou 2017].

However, sampling algorithms that access an infinite amount of entropy and compute with infinite precision real arithmetic cannot be implemented on physical machines. In practice, these algorithms are implemented on machines which use a finite amount of entropy and compute with approximate real arithmetic (e.g., double-precision floating point). As a result, sampling algorithms typically have a non-zero sampling error, which is challenging to systematically assess in practice [Devroye 1982]. ${ }^{2}$ While the quality of sampling algorithms implemented in practice is often characterized

\footnotetext{
${ }^{2}$ von Neumann [1951] objected that "the amount of theoretical information about the statistical properties of the round-off mechanism is nil" and, more humorously, that "anyone who considers arithmetic methods of producing random digits is, of course, in a state of sin."
} 
using ad-hoc statistical goodness-of-fit tests on a large number of simulations [Walker 1974; Leydold and Chaudhuri 2014], these empirical metrics fail to give rigorous statistical guarantees about the accuracy and/or theoretical optimality of the algorithm [Monahan 1985]. In this paper, we consider an alternative computational model that is more appropriate in applications where limited numerical precision, sampling error, or entropy consumption are of interest.

\subsection{The Random Bit Model}

In the random bit model, introduced by von Neumann [1951], the basic unit of randomness is a random symbol in the set $\{0,1, \ldots, b-1\}$ for some integer $b \geq 2$, obtained using a primitive called flip(). Since the random symbols are produced lazily by the source and the output of the sampling algorithm is a deterministic function of the discrete symbols, this model is suitable for analyzing entropy consumption and sampling error. In this paper, we consider the random bit model of computation where any sampling algorithm for a target distribution $\mathbf{p}$ over $[n]$ operates under the following assumptions:

A1. each invocation of $\mathrm{flip}()$ returns a single fair (unbiased) binary digit in $\{0,1\}$ (i.e., $b=2$ );

A2. the bits returned by separate invocations of flip() are all mutually independent;

A3. the output of the sampling algorithm is a single outcome in $[n]$, which is independent of all previous outputs of the algorithm; and

A4. the output probabilities of the sampling algorithm can be specified using at most $k$ binary digits, where the numerical precision parameter $k$ is specified independently of the target distribution $\mathbf{p}$.

Several limited-precision algorithms for sampling from discrete probability distributions in the literature operate under assumptions similar to A1-A4; examples include samplers for the uniform [Lumbroso 2013], discrete Gaussian [Folláth 2014], geometric [Bringmann and Friedrich 2013], random graph [Blanca and Mihail 2012], and general discrete [Uyematsu and Li 2003] distributions. Since these sampling algorithms use limited numerical precision that is specified independently of the target distribution (A4), they typically have some statistical sampling error.

We also note that several variants of the random bit model for random variate generation, which operate under different assumptions than A1-A4, have been thoroughly investigated in the literature. These variants include using a random source which provides flips of a biased $b$-sided coin (where the bias may be known or unknown); using a random source which provides non-i.i.d. symbols; sampling algorithms which return a random number of non-independent output symbols in each invocation; and/or sampling algorithms which use arithmetic operations whose numerical precision depends on the probabilities in the target distribution [von Neumann 1951; Elias 1972; Stout and Warren 1984; Blum 1986; Roche 1991; Peres 1992; Han and Verdú 1993; Vembu and Verdú 1995; Abrahams 1996; Pae and Loui 2006; Cicalese et al. 2006; Kozen 2014; Kozen and Soloviev 2018]. For example, Pae and Loui [2006] solve the very general problem of optimally simulating an arbitrary target distribution using $k$ independent flips of a $b$-sided coin with unknown bias, where optimality is defined in the sense of the asymptotic ratio of output bits per input symbol. Kozen and Soloviev [2018] provide a unifying coalgebraic framework for implementing and composing entropy-preserving reductions between arbitrary input sources to output distributions, describe several concrete algorithms for reductions between random processes, and present bounds on the trade-off between the latency and asymptotic entropy-efficiency of these protocols.

The assumptions A1-A4 that we make in this paper are designed to explore a new set of tradeoffs compared to those explored in previous works. More specifically, the current paper trades off accuracy with numerical precision in the non-asymptotic setting, while maintaining entropyoptimality of the output distribution, whereas the works of Pae and Loui [2006] and Kozen and 
Soloviev [2018], for example, trade off asymptotic entropy-efficiency with numerical precision, while maintaining perfect accuracy. The trade-offs we consider are motivated by the standard practice in numerical sampling libraries [Lea 1992; MathWorks 1993; R Core Team 2014; Galassi et al. 2019], which (i) use an entropy source that provides independent fair bits (modulo the fact that they may use pseudorandom number generators); (ii) implement samplers that guarantee exactly one output symbol per invocation; (iii) implement samplers that have non-zero output error; and (iv) use arithmetic systems with a fixed amount of precision (using e.g., 32-bit or 64-bit floating point). For the trade-offs considered in this paper, we present results that conclusively solve the problem of finding entropy-optimal sampling algorithms operating within any precision specification that yield closest-approximation distributions among the class of all entropy-optimal samplers that also operate within the given precision. The next section formalizes these concepts.

\subsection{Preliminaries}

Definition 2.1 (Sampling algorithm). Let $n \geq 1$ be an integer. A sampling algorithm, or sampler, $A: \biguplus_{k=1}^{\infty}\{0,1\}^{k} \rightarrow\{1, \ldots, n, \perp\}$ is a map that sends each finite tuple of bits to either an outcome in $[n]$ or a special symbol $\perp$ that indicates more bits are needed to determine the final outcome.

Remark 2.2. In Assumption A1 and Definition 2.1, the assumption that the source outputs binary digits in $\{0,1\}$ (i.e., $b=2$ ) is made without loss of generality. All the definitions and results in this paper generalize directly to the case of a source that outputs fair flips of any $b$-sided coin.

Knuth and Yao [1976] present a computational framework for expressing the set of all sampling algorithms for discrete probability distribution in the random bit model. Any sampling algorithm $A$ that draws random bits and returns an integer outcome $i$ with probability $p_{i}(i=1, \ldots, n)$ is equivalent to some (possibly infinite) binary tree $T$. Each internal node of $T$ has exactly 2 children and each leaf node is labeled with an outcome in $[n]$. The sampling algorithm starts at the root of $T$. It then draws a random bit $b$ from the source and takes the left branch if $b=0$ or the right branch if $b=1$. If the child node is a leaf node, the label assigned to that leaf is returned and the computation halts. Otherwise, the child node is an internal node, so a new random bit is drawn from the source and the process repeats. The next definition presents a state machine model that formally describes the behavior of any sampling algorithm in terms of such a computation tree.

Definition 2.3 (Discrete distribution generating tree). Let $A$ be a sampling algorithm. The computational behavior of $A$ is described by a state machine $T=(S, r, n, c, \delta)$, called the discrete distribution generating (DDG) tree of $A$, where

- $S \subseteq \mathbb{N}$ is a set of states (nodes);

- $r \in S$ is a designated start node;

- $n \geq 1$ is an integer indicating the number of outcomes of the sampler;

- $c: S \rightarrow\{1, \ldots, n\} \cup\{$ branch $\}$ is a function that labels each node as either a branch node or a terminal (leaf) node assigned to an outcome in $[n]$; and

- $\delta: S \times\{0,1\} \rightarrow S$ is a transition function that maps a node and a random bit to a new node.

Let $\mathbf{b}_{k}:=\left(b_{1}, \ldots, b_{k}\right) \in\{0,1\}^{k}$ be a tuple of $k \geq 0$ bits, $i \in S$ a state, and $j \in \mathbb{N}$. The operational semantics of $T$ for a configuration $\left\langle i, j, \mathbf{b}_{k}\right\rangle$ of the state machine are defined by the following rules

$$
\frac{0 \leq j<k ; c(i)=\text { branch }}{\left\langle i, j, \mathbf{b}_{k}\right\rangle_{T} \rightarrow\left\langle\delta\left(i, b_{j+1}\right), j+1, \mathbf{b}_{k}\right\rangle_{T}} \quad \frac{k \leq j ; c(i)=\text { branch }}{\left\langle i, j, \mathbf{b}_{k}\right\rangle_{T} \rightarrow \perp} \quad \frac{0 \leq j \leq k ; c(i) \in[n]}{\left\langle i, j, \mathbf{b}_{k}\right\rangle_{T} \rightarrow c(i)}
$$

In Eq. (2), the arrow $\rightarrow$ defines a transition relation from the current configuration (i.e., state $i$, consumed bits $j$, and input bits $\mathbf{b}_{k}$ ) to either a new configuration (first rule) or to a terminal outcome in $\{1, \ldots, n, \perp\}$ (second and third rules). The output of $A$ on input $\mathbf{b}_{k}$ is given by $A\left(\mathbf{b}_{k}\right):=\left\langle r, 0, \mathbf{b}_{k}\right\rangle_{T}$. 
Definition 2.4 (Output distribution). Let $T$ be the DDG tree of a sampler $A, 1[\cdot]$ the indicator function, and $\mathbf{b}_{k} \sim$ Uniform $\left(\{0,1\}^{k}\right)$ a random draw of $k \geq 0$ fair independent bits. Then

$$
\operatorname{Pr}\left[A\left(\mathbf{b}_{k}\right)=i\right]=\frac{1}{2^{k}} \sum_{\mathbf{b}^{\prime} \in\{0,1\}^{k}} 1\left[\left\langle\left(r, 0, \mathbf{b}^{\prime}\right)\right\rangle_{T}=i\right] \quad(i=1, \ldots, n) .
$$

The overall probability of returning $i$, over an infinite length random stream $b_{\infty}$ from the source, is

$$
p_{i}:=\operatorname{Pr}\left[A\left(\mathbf{b}_{\infty}\right)=i\right]=\lim _{k \rightarrow \infty} \operatorname{Pr}\left[A\left(\mathbf{b}_{k}\right)=i\right] \quad(i=1, \ldots, n) .
$$

For each $k$ we have $\operatorname{Pr}\left[A\left(\mathbf{b}_{k}\right)=\perp\right]=1-\sum_{i=1}^{n} \operatorname{Pr}\left[A\left(\mathbf{b}_{k}\right)=i\right]$. The list of outcome probabilities $\left(p_{1}, \ldots, p_{n}\right)$ defined in Eq. (4) is the called the output distribution of $T$, and we say that $T$ is wellformed whenever these probabilities sum to one (equivalently, whenever $A$ halts with probability one, so that $\left.\operatorname{Pr}\left[A\left(\mathbf{b}_{\infty}\right)=\perp\right]=0\right)$.

Definition 2.5 (Number of consumed bits). For each $k \geq 0$, let $\mathbf{b}_{k} \sim$ Uniform $\left(\{0,1\}^{k}\right)$ be a random draw of $k$ bits from the source. The number of bits consumed by $A$ is a random variable defined by

$$
N_{k}\left(A, \mathbf{b}_{k}\right):=\min \left(k, \min _{1 \leq j \leq k}\left\{j \mid A\left(b_{1}, \ldots, b_{j}\right) \in[n]\right\}\right) \quad(k=0,1, \ldots) .
$$

(where $\min (\varnothing):=\infty$ ), which is precisely the (random) number of steps executed in the evaluation rules (2) on the (random) input $\mathbf{b}_{k}$. Furthermore, we define $N(A):=\lim _{k \rightarrow \infty} N_{k}\left(A, \mathbf{b}_{k}\right)$ to be the limiting number of bits per sample, which exists (in the extended reals) whenever $T$ is well-formed

Definition 2.6 (Entropy [Shannon 1948]). Let $\mathbf{p}$ be a probability distribution over $[n]$. The Shannon entropy $H(\mathbf{p}):=\sum_{i=1}^{n} p_{i} \log \left(1 / p_{i}\right)$ is a measure of the stochasticity of $\mathbf{p}$ (unless otherwise noted, all instances of log are base 2). For each integer $n$, a deterministic distribution has minimal entropy $(H(\mathbf{p})=0)$ and the uniform distribution has maximal entropy $(H(\mathbf{p})=\log (n))$.

Definition 2.7 (Entropy-optimal sampler). A sampling algorithm A (or DDG tree T) with output distribution $\mathbf{p}$ is called entropy-optimal if the expected number of random bits consumed from the source is minimal among all samplers (or DDG trees) that yield the same output distribution p.

Definition 2.8 (Concise binary expansion). We say that a binary expansion of a rational number is concise if its repeating part is not of the form $\overline{1}$. In other words, to be concise, the binary expansions of dyadic rationals must end in $\overline{0}$ rather than $\overline{1}$.

Theorem 2.9 (KNUth AND YAo [1976]). Let $\mathbf{p}:=\left(p_{1}, \ldots, p_{n}\right)$ be a discrete probability distribution for some positive integer $n$. Let $A$ be an entropy-optimal sampler whose output distribution is equal to p. Then the number of bits $N(A)$ consumed by A satisfies $H(\mathbf{p}) \leq \mathbb{E}[N(A)]<H(\mathbf{p})+2$. Further, the underlying $D D G$ tree $T$ of $A$ contains exactly 1 leaf node labeled $i$ at level $j$ if and only if $p_{i j}=1$, where $\left(0 . p_{i 1} p_{i 2} \ldots\right)_{2}$ denotes the concise binary expansion of each $p_{i}$.

We next present three examples of target distributions and corresponding DDG trees that are both entropy-optimal, based on the construction from Theorem 2.9 and entropy-suboptimal. By Theorem 2.9, an entropy-optimal DDG tree for $\mathbf{p}$ can be constructed directly from a data structure called the binary probability matrix $\mathbf{P}$, whose entry $\mathrm{P}[i, j]$ corresponds to the $j$ th bit in the concise binary expansion of $p_{i}(i=1, \ldots, n ; j \geq 0)$. In general, the matrix $\mathbf{P}$ can contain infinitely many columns, but it can be finitely encoded when the probabilities of $\mathbf{p}$ are rational numbers.

In the case where each $p_{i}$ is dyadic, as in Example 2.10, we may instead work with the finite matrix $\mathbf{P}$ that omits those columns corresponding to a final $\overline{0}$ in every row, i.e., whose width is the maximum number of non-zero binary digits to the right of " 0. " in a concise binary expansion of $p_{i}$. 


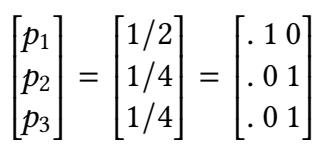

Binary probability matrix

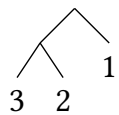

Entropy-optimal DDG tree

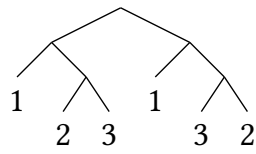

Entropy-suboptimal DDG tree

Example 2.10. Consider the distribution $\mathbf{p}:=(1 / 2,1 / 4,1 / 4)$ over $\{1,2,3\}$. Since $p_{1}=(0.10)_{2}$ and $p_{2}=p_{3}=(0.01)_{2}$ are all dyadic, the finite matrix $\mathbf{P}$ has two columns and the entropy-optimal tree has three levels (the root is level zero). Also shown above is an entropy-suboptimal tree for $\mathbf{p}$.

Now consider the case where the values of $\mathbf{p}$ are all rational but not all dyadic, as in Example 2.11. Then the full binary probability matrix can be encoded using a probability "pseudomatrix" $\mathbf{P}$, which has a finite number of columns that contain the digits in the finite prefix and the infinitely-repeating suffix of the concise binary expansions (a horizontal bar is placed atop the columns that contain the repeating suffix). Similarly, the infinite-level DDG tree for $\mathbf{p}$ can be finitely encoded by using backedges in a "pseudotree". Note that the DDG trees from Definition 2.3 are technically pseudotrees of this form, where $\delta$ encodes back-edges that finitely encode infinite trees with repeating structure. The terms "trees" and "pseudotrees" are used interchangeably throughout the paper.

$$
\left[\begin{array}{l}
p_{1} \\
p_{2}
\end{array}\right]=\left[\begin{array}{l}
3 / 10 \\
7 / 10
\end{array}\right]=\left[\begin{array}{llll}
0 & 0 \overline{10} & 0 & 1
\end{array}\right]
$$

Binary probability matrix

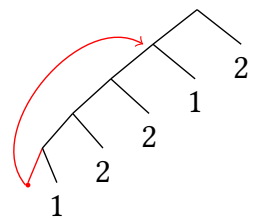

Entropy-optimal DDG tree

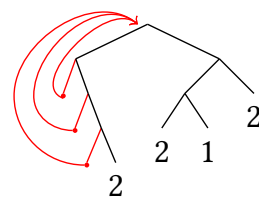

Entropy-suboptimal DDG tree

Example 2.11. Consider the distribution $\mathbf{p}:=(3 / 10,7 / 10)$ over $\{1,2\}$. As $p_{1}$ and $p_{2}$ are non-dyadic rational numbers, their infinite binary expansions can be finitely encoded using a pseudotree. The (shortest) entropy-optimal pseudotree shown above has five levels and a back-edge (red) from level four to level one. This structure corresponds to the structure of $\mathbf{P}$, which has five columns and a prefix length of one, as indicated by the horizontal bar above the last four columns of the matrix.

If any probability $p_{i}$ is irrational, as in Example 2.12, then its concise binary expansion will not repeat, and so we must work with the full binary probability matrix, which has infinitely many columns. Any DDG tree for $\mathbf{p}$ has infinitely many levels, and neither the matrix nor the tree can be finitely encoded. Probability distributions whose samplers cannot be finitely encoded are not the focus of the sampling algorithms in this paper.

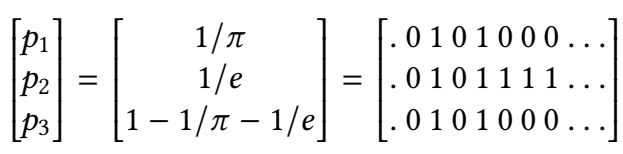

Binary probability matrix

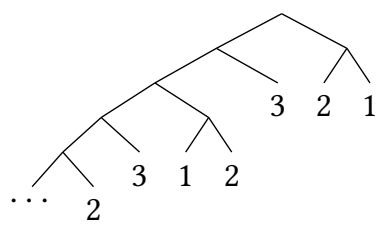

Entropy-optimal DDG tree

Example 2.12 (Knuth and Yao [1976]). Consider the distribution $\mathbf{p}:=(1 / \pi, 1 / e, 1-1 / \pi-1 / e)$ over $\{1,2,3\}$. The binary probability matrix has infinitely many columns and the corresponding DDG tree shown above has infinitely many levels, and neither can be finitely encoded. 


\subsection{Sampling Algorithms with Limited Computational Resources}

The previous examples present three classes of sampling algorithms, which are mutually exclusive and collectively exhaustive: Example 2.10 shows a sampler that halts after consuming at most $k$ bits from the source and has a finite DDG tree; Example 2.11 shows a sampler that needs an unbounded number of bits from the source and has an infinite DDG tree that can be finitely encoded; and Example 2.12 shows a sampler that needs an unbounded number of bits from the source and has an infinite DDG tree that cannot be finitely encoded. The algorithms presented in this paper do not consider target distributions and samplers that cannot be finitely encoded.

In practice, any sampler $A$ for a distribution $\mathbf{p}$ of interest that is implemented in a finite-resource system must correspond to a DDG tree $T$ with a finite encoding. As a result, the output probability of the sampler is typically an approximation to $\mathrm{p}$. This approximation arises from the fact that finite-resource machines do not have unbounded memory to store or even lazily construct DDG trees with an infinite number of levels-a necessary condition for perfectly sampling from an arbitrary target distribution-let alone construct entropy-optimal ones by computing the infinite binary expansion of each $p_{i}$. Even for a target distribution whose probabilities are rational numbers, the size of the entropy-optimal DDG tree may be significantly larger than the available resources on the system (Theorem 3.5). Informally speaking, a "limited-precision" sampler $A$ is able to represent each probability $p_{i}$ using no more than $k$ binary digits. The framework of DDG trees allows us to precisely characterize this notion in terms of the maximum depth of any leaf in the generating tree of $A$, which corresponds to the largest number of bits used to encode some $p_{i}$.

Definition 2.13 (Precision of a sampling algorithm). Let $A$ be any sampler and $T:=(S, r, n, c, \delta)$ its DDG tree. We say that $A$ uses $k$ bits of precision (or that $A$ is a $k$-bit sampler) if $S$ is finite and the longest simple path through $\delta$ starting from the root $r$ to any leaf node $l$ has exactly $k$ edges.

Remark 2.14. Suppose $A$ uses $k$ bits of precision. If $\delta$ is cycle-free, as in Example 2.10, then $A$ halts after consuming no more than $k$ bits from the source and has output probabilities that are dyadic rationals. If $\delta$ contains a back-edge, as in Example 2.11, then $A$ can consume an unbounded number of bits from the source and has output probabilities that are general rationals.

Given a target distribution $\mathbf{p}$, there may exist an exact sampling algorithm for $\mathbf{p}$ using $k$ bits of precision which is entropy-suboptimal and for which the entropy-optimal exact sampler requires $k^{\prime}>k$ bits of precision. Example 2.11 presents such an instance: the entropy-suboptimal DDG tree has depth $k=4$ whereas the entropy-optimal DDG tree has depth $k^{\prime}=5$. Entropy-suboptimal exact samplers typically require polynomial precision (in the number of bits used to encode $\mathbf{p}$ ) but can be slow and wasteful of random bits (Example 5.1), whereas entropy-optimal exact samplers are fast but can require precision that is exponentially large (Theorem 3.5). In light of these space-time trade-offs, this paper considers the problem of finding the "most accurate" entropy-optimal sampler for a target distribution $\mathbf{p}$ when the precision specification is set to a fixed constant (recall from Section 1 that fixing the precision independently of $\mathbf{p}$ necessarily introduces sampling error).

Problem 2.15. Given a target probability distribution $\mathbf{p}:=\left(p_{1}, \ldots, p_{n}\right)$, a measure of statistical error $\Delta$, and a precision specification of $k \geq 1$ bits, construct a $k$-bit entropy-optimal sampler $\hat{T}$ whose output probabilities $\hat{\mathbf{p}}$ achieve the smallest possible error $\Delta(\mathbf{p}, \hat{\mathbf{p}})$.

In the context of Problem 2.15, we refer to $\hat{\mathbf{p}}$ as a closest approximation to $\mathbf{p}$, or as a closestapproximation distribution to $\mathbf{p}$, and say that $\hat{T}$ is an optimal approximate sampler for $\mathbf{p}$.

For any precision specification $k$, the $k$-bit entropy-optimal samplers that yield some closest approximation to a given target distribution are not necessarily closer to $\mathbf{p}$ than all $k$-bit entropysuboptimal samplers. The next proposition, however, shows they obtain the smallest error among the class of all samplers that always halt after consuming at most $k$ random bits from the source. 
Proposition 2.16. Given a target $\mathbf{p}:=\left(p_{1}, \ldots, p_{n}\right)$, an error measure $\Delta$, and $k \geq 1$, suppose $\hat{T}$ is a $k$-bit entropy-optimal sampler whose output distribution is a $\Delta$-closest approximation to $\mathbf{p}$. Then $\hat{\mathbf{p}}$ is closer to $\mathbf{p}$ than the output distribution $\widetilde{\mathbf{p}}$ of any sampler $\widetilde{T}$ that halts after consuming at most $k$ random bits from the source.

Proof. Suppose for a contradiction that there is an approximation $\widetilde{\mathbf{p}}$ to $\mathbf{p}$ which is the output distribution of some sampler (either entropy-optimal or entropy-suboptimal) that consumes no more than $k$ bits from the source such that $\Delta(\mathbf{p}, \widetilde{\mathbf{p}})<\Delta(\mathbf{p}, \hat{\mathbf{p}})$. But then all entries in $\widetilde{\mathbf{p}}$ must be $k$-bit dyadic rationals. Thus, any entropy-optimal DDG tree $\widetilde{T}$ for $\widetilde{\mathbf{p}}$ has depth $k$ and no back-edges, contradicting the assumption that the output distribution $\hat{\mathbf{p}}$ of $\hat{T}$ is a closest approximation to $\mathbf{p}$.

Remark 2.17. In light of Proposition 2.16, we will also consider the restriction of Problem 2.15 to $k$-bit entropy-optimal samplers whose DDG trees do not have back-edges, which yields an entropy-optimal sampler in the class of samplers that halt after consuming at most $k$ random bits.

\subsection{Pitfalls of Naively Truncating the Target Probabilities}

Let us momentarily consider the class of samplers from Proposition 2.16. Namely, for given a precision specification $k$ and target distribution p, solve Problem 2.15 over the class of all algorithms that halt after consuming at most $k$ random bits (and thus have output distributions whose probabilities are dyadic rationals). This section shows examples of how naively truncating the target probabilities $p_{i}$ to have $k$ bits of precision (as in, e.g., Ladd [2009]; Dwarakanath and Galbraith [2014]) can fail to deliver accurate limited-precision samplers for various target distributions and error measures.

More specifically, the naive truncation initializes $\hat{p}_{i}=\left(0 . p_{1} p_{2} \ldots p_{k}\right)_{2}=\left\lfloor 2^{k} p_{i}\right\rfloor / 2^{k}$. As the $\hat{p}_{i}$ may not sum to unity, lower-order bits can be arbitrarily incremented until the terms sum to one (this normalization is implicit when using floating-point computations to implement limited-precision inversion sampling, as in Algorithm 2). The $\hat{p}_{i}$ can be organized into a probability matrix $\hat{\mathbf{P}}$, which is the truncation of the full probability matrix $\mathbf{P}$ to $k$ columns. The matrix $\hat{\mathbf{P}}$ can then be used to construct a finite entropy-optimal DDG tree, as in Example 2.10. While such a truncation approach may be sensible when the error of the approximate probabilities $\hat{p}_{i}$ is measured using total variation distance, the error in the general case can be highly sensitive to the setting of lower-order bits after truncation, depending on the target distribution $\mathbf{p}$, the precision specification $k$, and the error measure $\Delta$. We next present three conceptual examples that highlight these numerical issues for common measures of statistical error that are used in various applications.

Example 2.18 (Round-off with relative entropy divergence). Suppose the error measure is relative entropy (Kullback-Leibler divergence), $\Delta(\mathbf{p}, \hat{\mathbf{p}}):=\sum_{i=1}^{n} \log \left(\hat{p}_{i} / p_{i}\right) p_{i}$, which plays a key role in information theory and data compression [Kullback and Leibler 1951]. Suppose $n, k$ and $\mathbf{p}$ are such that $n \leq 2^{k}$ and there exists $i$ where $p_{i}=\epsilon \ll 1 / 2^{k}$. Then setting $\hat{p}_{i}$ so that $2^{k} \hat{p}_{i}=\left\lfloor 2^{k} p_{i}\right\rfloor=0$ and failing to increment the lower-order bit of $\hat{p}_{i}$ results in an infinite divergence of $\hat{\mathbf{p}}$ from $\mathbf{p}$, whereas, from the assumption that $n \leq 2^{k}$, there exist approximations that have finite divergence.

In the previous example, failing to increment a low-order bit results in a large (infinite) error. In the next example, choosing to increment a low-order bit results in an arbitrarily large error.

Example 2.19 (Round-off with Pearson chi-square divergence). Suppose the error measure is Pearson chi-square, $\Delta(\mathbf{p}, \hat{\mathbf{p}}):=\sum_{i=1}^{n}\left(p_{i}-\hat{p}_{i}\right)^{2} / p_{i}$, which is central to goodness-of-fit testing in statistics [Pearson 1900]. Suppose that $k$ and $\mathbf{p}$ are such that there exists $i$ where $p_{i}=c / 2^{k}+\epsilon$, for $0<\epsilon \ll 1 / 2^{k}$ for some integer $0 \leq c \leq 2^{k}-1$. Then setting $\hat{p}_{i}$ so that $2^{k} \hat{p}_{i}=\left\lfloor 2^{k} p_{i}\right\rfloor=c$ (not incrementing the lower-order bit) gives a small contribution to the error, whereas setting $\hat{p}_{i}^{+}$so that $2^{k} \hat{p}_{i}^{+}=\left\lfloor 2^{k} p_{i}\right\rfloor=c+1$ (incrementing the lower-order bit) gives a large contribution to the error. 
More specifically, the relative error of selecting $\hat{p}_{i}^{+}$instead of $\hat{p}_{i}$ is arbitrarily large:

$$
\begin{aligned}
\left(p_{i}-\hat{p}_{i}^{+}\right)^{2} /\left(p_{i}-\hat{p}_{i}\right)^{2} & =\left(c / 2^{k}+\epsilon-c / 2^{k}-1 / 2^{k}\right)^{2} /\left(c / 2^{k}+\epsilon-c / 2^{k}\right)^{2} \\
& =\left(1 / 2^{k}-\epsilon\right)^{2} / \epsilon^{2} \approx 1 /\left(2^{k} \epsilon\right)^{2} \gg 1 .
\end{aligned}
$$

The next example shows that the first $k$ bits of $p_{i}$ can be far from the globally optimal $k$-bit approximation, even in higher-precision regimes where $1 / 2^{k} \leq \min \left(p_{1}, \ldots, p_{n}\right)$.

Example 2.20 (Round-off with Hellinger divergence). Suppose the error measure is the Hellinger divergence, $\Delta(\mathbf{p}, \hat{\mathbf{p}}):=\sum_{i=1}^{n}\left(\sqrt{p_{i}}-\sqrt{\hat{p}_{i}}\right)^{2}$, which is used in fields such as information complexity [BarYossef et al. 2004]. Let $k=16$ and $n=1000$, with $p_{1}=5 / 8$ and $p_{2}=\cdots=p_{n}=3 / 8(n-1)$. Let $\left(\hat{p}_{1}, \ldots \hat{p}_{n}\right)$ be the $k$-bit approximation that minimizes $\Delta(\mathbf{p}, \hat{\mathbf{p}})$. It can be shown that $2^{k} \hat{p}_{1}=40788$ whereas $\left\lfloor 2^{k} p_{1}\right\rfloor=40960$, so that $\left|\left\lfloor 2^{k} p_{1}\right\rfloor-2^{k} \hat{p}_{1}\right|=172$.

In light of these examples, we turn our attention to solving Problem 2.15 by truncating the target probabilities in a principled way that avoids these pitfalls and finds a closest-approximation distribution for any target probability distribution, error measure, and precision specification.

\section{CHARACTERIZING THE SPACE OF ENTROPY-OPTIMAL SAMPLING ALGORITHMS}

This section presents several results about the class of entropy-optimal $k$-bit sampling algorithms over which Problem 2.15 is defined. These results form the basis of the algorithm for finding a closestapproximation distribution $\hat{\mathbf{p}}$ in Section 4 and the algorithms for constructing the corresponding entropy-optimal DDG tree $\hat{T}$ in Section 5, which together will form the solution to Problem 2.15.

Section 2.4 considered sampling algorithms that halt after consuming at most $k$ random bits (so that each output probability is an integer multiple of $1 / 2^{k}$ ) and showed that naively discretizing the target distribution can result in poor approximations. The DDG trees of those sampling algorithms are finite: they have depth $k$ and no back-edges. For entropy-optimal DDG trees that use $k \geq 1$ bits of precision (Definition 2.13) and have back-edges, the output distributions (Definition 2.4) are described by a $k$-bit number. The $k$-bit numbers $x$ are those such that for some integer $l$ satisfying $0 \leq l \leq k$, there is some element $\left(x_{1}, \ldots, x_{k}\right) \in\{0,1\}^{l} \times\{0,1\}^{k-l}$, where the first $l$ bits correspond to a finite prefix and the final $k-l$ bits correspond to an infinitely repeating suffix, such that $x=\left(0 . x_{1} \ldots x_{l} \overline{x_{l+1} \ldots x_{k}}\right)_{2}$. Write $\mathbb{B}_{k l}$ for the set of rationals in $[0,1]$ describable in this way.

Proposition 3.1. For integers $k$ and $l$ with $0 \leq l \leq k$, define $Z_{k l}:=2^{k}-2^{l} 1_{l<k}$. Then

$$
\mathbb{B}_{k l}=\left\{\frac{0}{Z_{k l}}, \frac{1}{Z_{k l}}, \ldots, \frac{Z_{k l}-1}{Z_{k l}}, \frac{Z_{k l}}{Z_{k l}} 1_{l<k}\right\} .
$$

Proof. For $l=k$, the number system $\mathbb{B}_{k l}=\mathbb{B}_{k k}$ is the set of dyadic rationals in [0,1) with denominator $Z_{k k}=2^{k}$. For $0 \leq l<k$, any element $x \in \mathbb{B}_{k l}$ when written in base 2 has a (possibly empty) non-repeating prefix and a non-empty infinitely repeating suffix, so that $x$ has binary expansion $\left(0 . a_{1} \ldots a_{l} \overline{s_{l+1} \ldots s_{k}}\right)_{2}$. The first two lines of equalities (Eqs. (7) and (8)) imply Eq. (9):

$$
\begin{aligned}
2^{l}\left(0 . a_{1} \ldots a_{l}\right)_{2} & =\left(a_{1} \ldots a_{l}\right)_{2}=\sum_{i=0}^{l-1} a_{l-i} 2^{i}, \\
\left(2^{k-l}-1\right)\left(0 . \overline{s_{l+1} \ldots s_{k}}\right)_{2} & =\left(s_{l+1} \ldots s_{k}\right)_{2}=\sum_{i=0}^{k-(l+1)} s_{k-i} 2^{i}, \\
x=\left(0 . a_{1} \ldots a_{l}\right)_{2}+2^{-l}\left(0 . \overline{s_{l+1} \ldots s_{k}}\right)_{2} & =\frac{\left(2^{k-l}-1\right) \sum_{i=0}^{l-1} a_{l-i} 2^{i}+\sum_{i=0}^{k-(l+1)} s_{k-i} 2^{i}}{2^{k}-2^{l}} .
\end{aligned}
$$

Remark 3.2. For a rational $x \in[0,1]$, we take a representative $\left(\left(x_{1}, \ldots, x_{l}\right),\left(x_{l+1}, \ldots, x_{k}\right)\right) \in \mathbb{B}_{k l}$ that is both concise (Definition 2.8) and chosen such that the number $k$ of digits is as small possible. 
Remark 3.3. When $0 \leq l \leq k$, we have $\mathbb{B}_{k l} \subseteq \mathbb{B}_{k+1, l+1}$, since if $x \in \mathbb{B}_{k l}$ then Proposition 3.1 furnishes an integer $c$ such that $x=c /\left(2^{k}-2^{l} \mathbf{1}_{l<k}\right)=2 c /\left(2^{k+1}-2^{l+1} \mathbf{1}_{l<k}\right) \in \mathbb{B}_{k+1, l+1}$. Further, for $k \geq 2$, we have $\mathbb{B}_{k, k-1} \backslash\{1\}=\mathbb{B}_{k-1, k-1} \subseteq \mathbb{B}_{k k}$, since any infinitely repeating suffix comprised of a single digit can be folded into the prefix, except when the prefix and suffix are all ones.

TheOREM 3.4. Let $\mathbf{p}:=\left(p_{1}, \ldots, p_{n}\right)$ be a non-degenerate rational distribution for some integer $n>1$. The precision $k$ of the shortest entropy-optimal DDG (pseudo)tree with output distribution $\mathbf{p}$ is the smallest integer such that every $p_{i}$ is an integer multiple of $1 / Z_{k l}$ (hence in $\mathbb{B}_{k l}$ ) for some $l \leq k$.

Proof. Suppose that $T$ is a shortest entropy-optimal DDG (pseudo)tree and let $k$ be its depth (note that $k \geq 1$, as $k=0$ implies $\mathrm{p}$ is degenerate). Assume $n=2$. From Theorem 2.9, Definition 2.13, and the hypothesis that the transition function $\delta$ of $T$ encodes that shortest possible DDG tree, we have that for each $i=1,2$, the probability $p_{i}$ is a rational number where the number of digits in the shortest prefix and suffix of the concise binary expansion is at most $k$. Therefore, we can write

$$
p_{1}=\left(0 . a_{1} \ldots a_{l_{1}} \overline{s_{l_{1}+1} \ldots s_{k}}\right), \quad p_{2}=\left(0 . w_{1} \ldots w_{l_{2}} \overline{u_{l_{2}+1} \ldots u_{k}}\right),
$$

where $l_{i}$ and $k-l_{i}$ are the number of digits in the shortest prefix and suffix, respectively, of each $p_{i}$.

If $l_{1}=l_{2}$ then the conclusion follows from Proposition 3.1. If $l_{1}=k-1$ and $l_{2}=k$ then the conclusion follows from Remark 3.3 and the fact that $p_{1} \neq 1, p_{2} \neq 1$. Now, from Proposition 3.1, it suffices to establish that $l_{1}=l_{2}=: l$, so that $p_{1}$ and $p_{2}$ are both integer multiples of $1 / Z_{k l}$. Suppose for a contradiction that $l_{1}<l_{2}$ and $l_{1} \neq k-1$. Write $p_{1}=a / c$ and $p_{2}=b / d$ where each summand is in reduced form. By Proposition 3.1, we have $c=2^{k}-2^{l_{1}}$ and $d=2^{k}-2^{l_{2}} \mathbf{1}_{l_{2}<k}$. Then as $p_{1}+p_{2}=1$ we have $a d+b c=c d$. If $c \neq d$ then either $b$ has a positive factor in common with $d$ or $a$ with $c$, contradicting the summands being in reduced form. But $c=d$ contradicts $l_{1}<l_{2}$.

The case where $n>2$ is a straightforward extension of this argument.

An immediate consequence of Theorem 3.4 is that all back-edges in an entropy-optimal DDG tree that uses $k$ bits of precision must originate at level $k-1$ and end at the same level $l<k-1$. The next result, Theorem 3.5, shows that at most $Z-1$ bits of precision are needed by an entropy-optimal DDG tree to exactly flip a coin with rational probability $p=c / Z$, which is exponentially larger than the $\log (Z)$ bits needed to encode $Z$. Theorem 3.6 shows that this bound is tight for many $Z$ and, as we note in Remark 3.7, is likely tight for infinitely many $Z$. These results highlight the need for approximate entropy-optimal sampling from a computational complexity standpoint.

Theorem 3.5. Let $M_{1}, \ldots, M_{n}$ be $n$ positive integers that sum to $Z$ and let $\mathbf{p}:=\left(M_{1} / Z, \ldots, M_{n} / Z\right)$. Any exact, entropy-optimal sampler whose output distribution is $\mathbf{p}$ needs at most $Z-1$ bits of precision.

Proof. By Theorem 3.4, it suffices to find integers $k \leq Z-1$ and $l \leq k$ such that $Z_{k l}$ is a multiple of $Z$, which in turn implies that any entropy-optimal sampler for p needs at most $Z-1$ bits.

Case 1: $Z$ is odd. Consider $k=Z-1$. We will show that $Z$ divides $2^{Z-1}-2^{l}$ for some $l$ such $0 \leq l \leq Z-2$. Let $\phi$ be Euler's totient function, which satisfies $1 \leq \phi(Z) \leq Z-1=k$. Then $2^{\phi(Z)} \equiv 1$ $(\bmod Z)$ as $\operatorname{gcd}(Z, 2)=1$. Put $l=Z-1-\phi(Z)$ and conclude that $Z$ divides $2^{Z-1}-2^{Z-1-\phi(Z)}$.

Case 2: $Z$ is even. Let $t \geq 1$ be the maximal power of 2 dividing $Z$, and write $Z=Z^{\prime} 2^{t}$. Consider $k=Z^{\prime}-1+t$ and $l=j+t$ where $j=\left(Z^{\prime}-1\right)-\phi\left(Z^{\prime}\right)$. As in the previous case applied to $Z^{\prime}$, we have that $Z^{\prime}$ divides $2^{Z^{\prime}-1}-2^{j}$, and so $Z$ divides $2^{k}-2^{l}$. We have $0 \leq l \leq k$ as $1 \leq \phi(Z) \leq Z-1$. Finally, $k=Z^{\prime}+t-1 \leq Z^{\prime} 2^{t}-1=Z-1$ as $t<2^{t}$.

Theorem 3.6. Let $M_{1}, \ldots, M_{n}$ be $n$ positive integers that sum to $Z$ and put $\mathbf{p}=\left(M_{1} / Z, \ldots, M_{n} / Z\right)$. If $Z$ is prime and 2 is a primitive root modulo $Z$, then any exact, entropy-optimal sampler whose output distribution is $\mathbf{p}$ needs exactly $Z-1$ bits of precision. 
Proof. Since 2 is a primitive root modulo $Z$, the smallest integer $a$ for which $2^{a}-1 \equiv 0(\bmod Z)$ is precisely $\phi(Z)=Z-1$. We will show that for any $k^{\prime}<Z-1$ there is no exact entropy-optimal sampler that uses $k^{\prime}$ bits of precision. By Theorem 3.5, if there were such a sampler, then $Z_{k^{\prime} l}$ must be a multiple of $Z$ for some $l \leq k^{\prime}$. If $l<k^{\prime}$, then $Z_{k^{\prime} l}=2^{k^{\prime}}-2^{l}$. Hence $2^{k^{\prime}} \equiv 2^{l}(\bmod Z)$ and so $2^{k^{\prime}-l} \equiv 1(\bmod Z)$ as $Z$ is odd. But $k^{\prime}<Z-1=\phi(Z)$, contradicting the assumption that 2 is a primitive root modulo $Z$. If $l=k^{\prime}$, then $Z_{k^{\prime} l}=2^{k^{\prime}}$, which is not divisible by $Z$ since we have assumed that $Z$ is odd (as 2 is not a primitive root modulo 2).

Remark 3.7. The bound in Theorem 3.5 is likely the tightest possible for infinitely many $Z$. Assuming Artin's conjecture, there are infinitely many primes $Z$ for which 2 is a primitive root, which in turns implies by Theorem 3.6 that any entropy-optimal DDG tree must have $Z$ levels.

\section{OPTIMAL APPROXIMATIONS OF DISCRETE PROBABILITY DISTRIBUTIONS}

Returning to Problem 2.15, we next present an efficient algorithm for finding a closest-approximation distribution $\hat{\mathbf{p}}$ to any target distribution p, using Theorem 3.4 to constrain the set of allowable distributions to those that are the output distribution of some entropy-optimal $k$-bit sampler.

\section{1 $f$-divergences: A Family of Statistical Divergences}

We quantify the error of approximate sampling algorithms using a broad family of statistical error measures called $f$-divergences [Ali and Silvey 1966], as is common in the random variate generation literature [Cicalese et al. 2006]. This family includes well-known divergences such as total variation (which corresponds to Euclidean $L_{1}$ norm), relative entropy (used in information theory [Kullback and Leibler 1951]), Pearson chi-square (used in statistical hypothesis testing [Pearson 1900]), Jensen-Shannon (used in text classification [Dhillon et al. 2003]), and Hellinger (used in cryptography [Steinberger 2012] and information complexity [Bar-Yossef et al. 2004]).

Definition 4.1 (Statistical divergence). Let $n$ be a positive integer and $\mathcal{S}_{n}$ be the $(n-1)$-dimensional probability simplex, i.e., the set of all probability distributions over $[n]$. A statistical divergence $\Delta: \mathcal{S}_{n} \times \mathcal{S}_{n} \rightarrow[0, \infty]$ is any mapping from pairs of distributions on $[n]$ to non-negative extended real numbers, such that for all $\mathbf{p}, \mathbf{q} \in \mathcal{S}_{n}$ we have $\Delta(\mathbf{p}, \mathbf{q})=0$ if and only if $p_{i}=q_{i}(i=1, \ldots, n)$.

Table 1. Common statistical divergences expressed as $f$-divergences.

\begin{tabular}{llc}
\hline Divergence Measure & Formula $\Delta_{g}(\mathbf{p}, \mathbf{q})$ & Generator $g(t)$ \\
\hline Total Variation & $\frac{1}{2} \sum_{i=1}^{n}\left|q_{i}-p_{i}\right|$ & $\frac{1}{2}|t-1|$ \\
Hellinger Divergence & $\frac{1}{2} \sum_{i=1}^{n}\left(\sqrt{p_{i}}-\sqrt{q_{i}}\right)^{2}$ & $(\sqrt{t}-1)^{2}$ \\
Pearson Chi-Squared & $\sum_{i=1}^{n}\left(q_{i}-p_{i}\right)^{2} / q_{i}$ & $(t-1)^{2}$ \\
Triangular Discrimination & $\sum_{i=1}^{n}\left(p_{i}-q_{i}\right)^{2} /\left(p_{i}+q_{i}\right)$ & $(t-1)^{2} /(t+1)$ \\
Relative Entropy & $\sum_{i=1}^{n} \log \left(q_{i} / p_{i}\right) q_{i}$ & $t \log t$ \\
$\alpha$-Divergence & $4\left(1-\sum_{i=1}^{n}\left(p_{i}^{(1-\alpha) / 2} q_{i}^{1+\alpha}\right)\right) /\left(1-\alpha^{2}\right)$ & $4\left(1-t^{(1+\alpha) / 2}\right) /\left(1-\alpha^{2}\right)$ \\
\hline
\end{tabular}

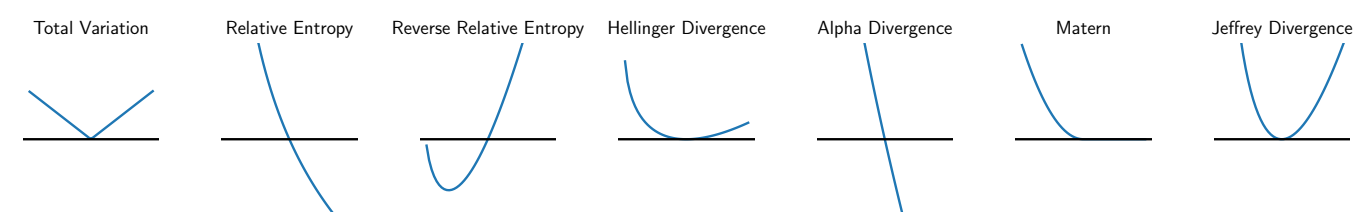

Fig. 1. Plots of generating functions $g$ for various $f$-divergences, a subset of which are shown in Table 1 . 
Definition 4.2 ( $f$-divergence). An $f$-divergence is any statistical divergence of the form

$$
\Delta_{g}(\mathbf{p}, \mathbf{q}):=\sum_{i=1}^{n} g\left(q_{i} / p_{i}\right) p_{i},
$$

for some convex function $g:(0, \infty) \rightarrow \mathbb{R}$ with $g(1)=0$. The function $g$ is called the generator of $\Delta_{g}$.

For concreteness, Table 1 expresses several statistical divergence measures as $f$-divergences and Figure 1 shows plots of generating functions. The class of $f$-divergences is closed under several operations; for example, if $\Delta_{g}(\mathbf{p}, \mathbf{q})$ is an $f$-divergence then so is the dual $\Delta_{g_{*}}(\mathbf{q}, \mathbf{p})$, where $g_{*}(t)=t g(1 / t)$ is the perspective of $g$. A technical review of these concepts can be found in Liese and Vajda [2006, Section III]. In this paper, we address Problem 4.6 assuming the error measure $\Delta$ is an $f$-divergence, which in turn allows us to optimize any error measure that is a 1-1 transformation of an underlying $f$-divergence.

\subsection{Problem Statement for Finding Closest-Approximation Distributions}

Recall that Theorem 3.4 establishes that the probability distributions that can be simulated exactly by an entropy-optimal DDG tree with $k$ bits of precision have probabilities $p_{i}$ of the form $M_{i} / Z_{k l}$, where $M_{i}$ is a non-negative integer and $Z_{k l}=2^{k}-2^{l} \mathbf{1}_{k<l}$ is the denominator of the number system $\mathbb{B}_{k l}$. This notion is a special case of the following concept.

Definition 4.3 (Z-type distribution [Cover and Thomas 2006]). For any positive integer $Z$, a probability distribution $\mathbf{p}$ over $[n]$ is said to be $Z$-type distribution if

$$
p_{i} \in\left\{\frac{0}{Z}, \frac{1}{Z}, \frac{2}{Z}, \ldots, \frac{Z}{Z}\right\} \quad(i=1, \ldots, n) .
$$

Definition 4.4. For positive integer $n$ and non-negative integer $Z$, define the set

$$
\mathcal{M}[n, Z]:=\left\{\left(M_{1}, \ldots, M_{n}\right) \mid M_{i} \geq 0, M_{i} \in \mathbb{Z}, \sum_{i=1}^{n} M_{i}=Z\right\},
$$

which can be thought of as the set of all possible assignments of $Z$ indistinguishable balls into $n$ distinguishable bins such that each bin $i$ has $M_{i}$ balls.

Remark 4.5. Each element $\mathbf{M} \in \mathcal{M}[n, Z]$ may be identified with a $Z$-type distribution $\mathbf{q}$ over $[n]$ by letting $q_{i}:=M_{i} / Z(i=1, \ldots, n)$, and thus adopt the notation $\Delta_{g}(\mathbf{p}, \mathbf{M})$ to indicate the $f$-divergence between probability distributions p and q (cf. Eq. (11)).

By Theorem 3.4 and Remark 4.5, Problem 2.15 is a special case of the following problem, since the output distribution of any $k$-bit entropy-optimal sampler is $Z$-type, where $Z \in\left\{Z_{k 0}, \ldots, Z_{k k}\right\}$.

Problem 4.6. Given a target distribution $\mathrm{p}$ over $[n]$, an $f$-divergence $\Delta_{g}$, and a positive integer $Z$, find a tuple $\mathbf{M}=\left(M_{1}, \ldots, M_{n}\right) \in \mathcal{M}[n, Z]$ that minimizes the divergence

$$
\Delta_{g}(\mathbf{p}, \mathbf{M})=\sum_{i=1}^{n} g\left(\frac{M_{i}}{Z p_{i}}\right) p_{i}
$$

As the set $\mathcal{M}[n, Z]$ is combinatorially large, Problem 4.6 cannot be solved efficiently by enumeration. In the next section, we present an algorithm that finds an assignment $\mathbf{M}$ that minimizes the objective function (14) among the elements of $\mathcal{M}[n, Z]$. By Theorem 3.4, for any precision specification $k \geq 0$, using $Z=Z_{k l}$ for each $l=0, \ldots, k$ and then selecting the value of $l$ for which Eq. (14) is smallest corresponds to finding a closest-approximation distribution $\hat{\mathbf{p}}$ for the class of $k$-bit entropy-optimal samplers, and thus solves the first part of Problem 2.15. 


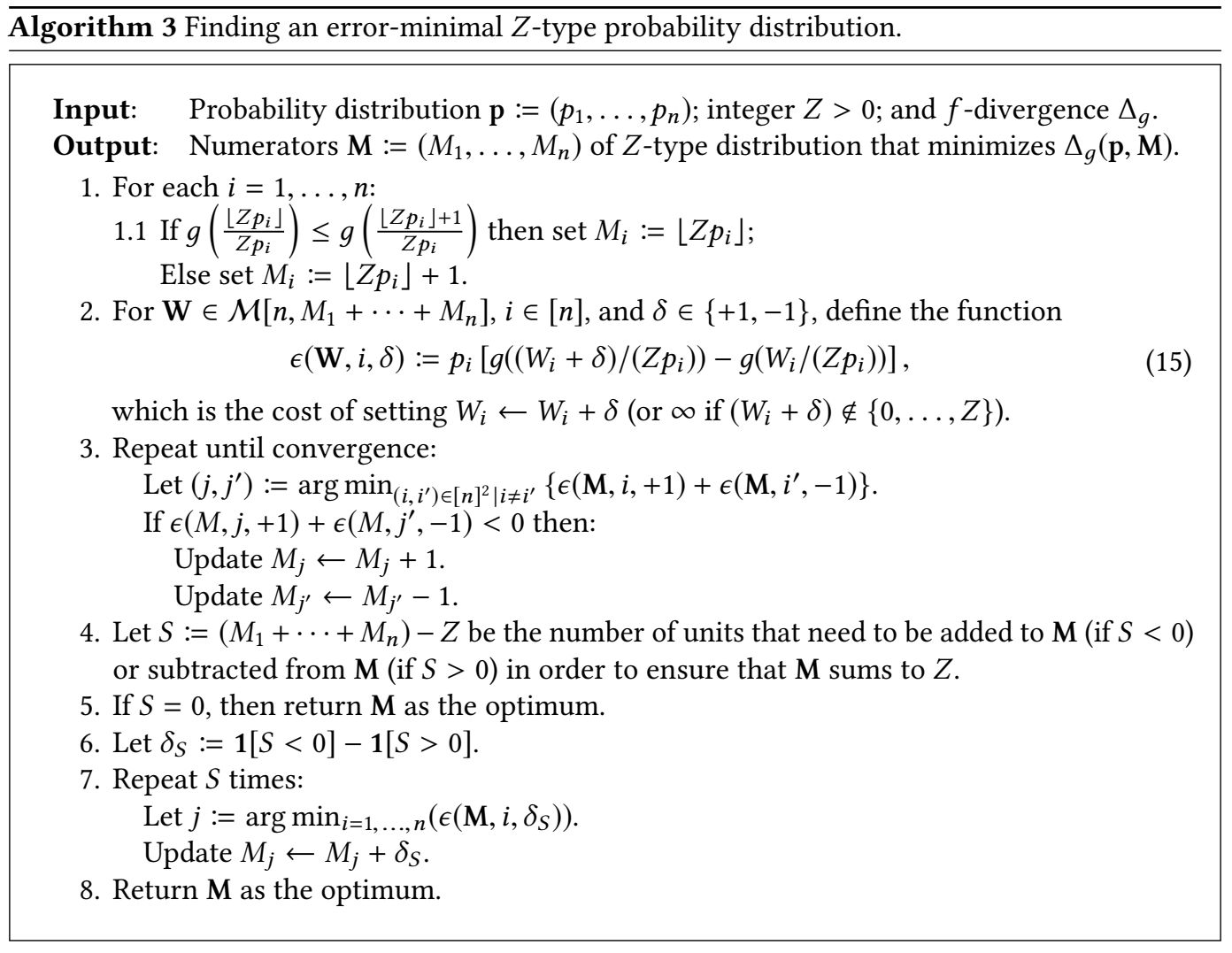

\subsection{An Efficient Optimization Algorithm}

Algorithm 3 presents an efficient procedure that solves Problem 4.6. We now state the main theorem.

Theorem 4.7. For any probability distribution $\mathbf{p}, f$-divergence $\Delta_{g}$, and denominator $Z>0$, the distribution returned by Algorithm 3 minimizes the objective function (14) over all $Z$-type distributions.

The remainder of this section contains the proof of Theorem 4.7. Section 4.3.1 establishes correctness and Section 4.3.2 establishes runtime.

4.3.1 Theoretical Analysis: Correctness. In this section, let $\mathbf{p}, n, Z$, and $g$ be defined as in Algorithm 3 .

Definition 4.8. Let $t>0$ be an integer and $\mathbf{M} \in \mathcal{M}[n, t]$. For integers $a$ and $b$, define

$$
\Delta^{i}[a \rightarrow b ; \mathbf{M}, Z]:=p_{i}\left[g\left(\frac{M_{i}+b}{Z p_{i}}\right)-g\left(\frac{M_{i}+a}{Z p_{i}}\right)\right] \quad(i=1, \ldots, n) .
$$

For typographical convenience, we write $\Delta^{i}[a \rightarrow b ; \mathbf{M}]$ (or $\Delta^{i}[a \rightarrow b]$ ) when $Z$ (and $\mathbf{M}$ ) are clear from context. We define $\Delta^{i}[a \rightarrow b]:=\infty$ whenever $\left(M_{i}+b\right)$ or $\left(M_{i}+a\right)$ are not in $\{0, \ldots, t\}$.

Remark 4.9. The convexity of $g$ implies that for any real number $j$,

$$
\frac{g\left(\frac{M_{i}+j+1}{Z p_{i}}\right)-g\left(\frac{M_{i}+j}{Z p_{i}}\right)}{1 /\left(Z p_{i}\right)} \leq \frac{g\left(\frac{M_{i}+j+2}{Z p_{i}}\right)-g\left(\frac{M_{i}+j+1}{Z p_{i}}\right)}{1 /\left(Z p_{i}\right)} \quad(i=1, \ldots, n) .
$$


Letting $j$ range over the integers gives

$$
\cdots<\Delta^{i}[-2 \rightarrow-1]<\Delta^{i}[-1 \rightarrow 0]<\Delta^{i}[0 \rightarrow 1]<\Delta^{i}[1 \rightarrow 2]<\Delta^{i}[2 \rightarrow 3]<\cdots .
$$

By telescoping (18), if $a<b<c$ then

$$
\Delta^{i}[a \rightarrow b]<\Delta^{i}[a \rightarrow c] .
$$

Finally, it is immediate from the definition that $\Delta^{i}[a \rightarrow b]=-\Delta^{i}[b \rightarrow a]$ for all $a$ and $b$.

THEOREM 4.10. Let $t>0$ be an integer and $\mathbf{M}:=\left(M_{1}, \ldots, M_{n}\right)$ be any assignment in $\mathcal{M}[n, t]$. If, given initial values $\mathbf{M}$ the loop defined in Step 3 of Algorithm 3 terminates, then the final values of $\mathbf{M}$ minimize $\Delta_{g}(\mathrm{p}, \cdot)$ over the set $\mathcal{M}[n, t]$.

Proof. We argue that the locally optimal assignments performed at each iteration of the loop are globally optimal. Assume toward a contradiction that the loop in Step 3 terminates with a suboptimal assignment $\left(W_{1}, \ldots, W_{n}\right) \in \mathcal{M}[n, t]$. Then there exist indices $i$ and $j$ with $1 \leq i<j \leq n$ such that for some positive integers $a$ and $b$,

$$
\begin{aligned}
p_{j} g\left(\frac{W_{j}+a}{Z p_{j}}\right)+p_{i} g\left(\frac{W_{i}-b}{Z p_{j}}\right) & <p_{j} g\left(\frac{W_{j}}{Z p_{j}}\right)+p_{i} g\left(\frac{W_{i}}{Z p_{j}}\right) \\
\Longleftrightarrow \Delta^{j}[0 \rightarrow a]+\Delta^{i}[0 \rightarrow-b] & <0 \\
& \Longleftrightarrow \Delta^{j}[0 \rightarrow a]<-\Delta^{i}[0 \rightarrow-b] \\
& \Longleftrightarrow \Delta^{j}[0 \rightarrow a]<\Delta^{i}[-b \rightarrow 0] .
\end{aligned}
$$

Combining (23) with (19) gives

$$
\Delta^{j}[0 \rightarrow 1]<\Delta^{j}[0 \rightarrow a]<\Delta^{i}[-b \rightarrow 0]<\Delta^{i}[-1 \rightarrow 0],
$$

which implies $\epsilon_{j}(+1)+\epsilon_{i}(-1)<0$, and so the loop can execute for one more iteration.

We now show that the value of $\mathbf{M}$ at the termination of the loop in Step 7 of Algorithm 3 optimizes the objective function over $\mathcal{M}[n, Z]$.

THeorem 4.11. For some positive integer $t<Z$, suppose that $\mathbf{M}:=\left(M_{1}, \ldots, M_{n}\right)$ minimizes the objective function $\Delta_{g}(\mathrm{p}, \cdot)$ over the set $\mathcal{M}[n, t]$. Then $\mathrm{M}^{+}$defined by $M_{i}^{+}:=M_{i}+\mathbf{1}_{i=u}$ minimizes $\Delta_{g}(\mathbf{p}, \cdot)$ over $\mathcal{M}[n, t+1]$, where

$$
u:=\underset{i=1, \ldots, n}{\arg \min }\left\{p_{i}\left[g\left(\frac{M_{i}+1}{Z p_{i}}\right)-g\left(\frac{M_{i}}{Z p_{i}}\right)\right]\right\} .
$$

Proof. Assume, for a contradiction, that there exists $\mathbf{M}^{\prime}:=\left(M_{1}^{\prime}, \ldots, M_{n}^{\prime}\right)$ that minimizes $\Delta_{g}(\mathbf{p}, \cdot)$ over $\mathcal{M}[n, t+1]$ with $\Delta_{g}\left(\mathbf{p}, \mathbf{M}^{\prime}\right)<\Delta_{g}\left(\mathbf{p}, \mathbf{M}^{+}\right)$. Clearly $\mathbf{M}^{\prime} \neq \mathbf{M}^{+}$. We proceed in cases.

Case 1: $M_{u}^{\prime}=M_{u}$. Then there exists integers $j \neq t$ and $a \geq 1$ such that $M_{j}^{\prime}=M_{j}+a$. Hence

$$
\begin{gathered}
\Delta^{u}[0 \rightarrow 1]<\Delta^{j}[0 \rightarrow 1] \leq \Delta^{j}[(a-1) \rightarrow a]=-\Delta^{j}[a \rightarrow(a-1)] \\
\Longrightarrow \Delta^{u}[0 \rightarrow 1]+\Delta^{j}[a \rightarrow(a-1)]<0,
\end{gathered}
$$

where the first inequality of (26) follows from the minimality of $u$ in (25) and the second inequality of (26) follows from (18). Therefore, setting $M_{u}^{\prime} \leftarrow M_{u}+1$ and $M_{j}^{\prime} \leftarrow M_{j}+(a-1)$ gives a net reduction in the cost, a contradiction to the optimality of $\mathbf{M}^{\prime}$.

Case 2: $M_{u}^{\prime}=M_{u}+1$. Assume without loss of generality (for this case) that $u=1$. Since $\mathbf{M}^{\prime} \neq \mathbf{M}^{+}$, there exists an index $j>1$ such that $M_{j}^{\prime} \neq M_{j}$. There are $t+1-\left(M_{1}+1\right)=t-M_{1}$ remaining units to distribute among $\left(M_{2}^{\prime}, \ldots, M_{n}^{\prime}\right)$. From the optimality of $\mathbf{M}$, the tail $\left(M_{2}, \ldots, M_{n}\right)$ minimizes $\sum_{i=2}^{n} p_{i} g\left(M_{i} / Z p_{i}\right)$ among all tuples using $t-M_{1}$ units; otherwise a more optimal solution could be 
obtained by holding $M_{1}$ fixed and optimizing $\left(M_{2}, \ldots, M_{n}\right)$. It follows that the tail $\left(M_{2}^{\prime}, \ldots, M_{n}^{\prime}\right)$ of $\mathbf{M}^{\prime}$ is less optimal than the tail $\left(M_{2}, \ldots, M_{n}\right)$ of $\mathbf{M}^{+}$, a contradiction to the optimality of $\mathbf{M}^{\prime}$.

Case 3: $M_{u}^{\prime}=M_{u}+c$ for some integer $c \geq 2$. Then there exists some $j \neq t$ such that $M_{j}^{\prime}=M_{j}-a$ for some integer $a \geq 1$. From the optimality of $\mathbf{M}$, any move must increase the objective, i.e.,

$$
\Delta^{u}[0 \rightarrow 1]>\Delta^{j}[-1 \rightarrow 0] .
$$

Combining (18) with (28) gives

$$
\begin{array}{r}
\Delta^{u}[(c-1) \rightarrow c] \geq \Delta^{u}[0 \rightarrow 1]>\Delta^{j}[-1 \rightarrow 0] \geq \Delta^{j}[-a \rightarrow-(a-1)] \\
\Longrightarrow \Delta^{u}[c \rightarrow(c-1)]+\Delta^{j}[-a \rightarrow-(a-1)]<0
\end{array}
$$

Therefore, setting $M_{u}^{\prime} \leftarrow M_{u}+(c-1)$ and $M_{j}^{\prime} \leftarrow M_{j}-(a-1)$ gives a net reduction in the cost, a contradiction to the optimality of $\mathbf{M}^{\prime}$.

Case 4: $M_{u}^{\prime}=M_{u}-a$ for some integer $a \geq 1$. This case is symmetric to the previous one.

By a proof symmetric to that of Theorem 4.11, we obtain the following.

Corollary 4.12. If $\mathbf{M}$ minimizes $\Delta_{g}(\mathbf{p}, \cdot)$ over $\mathcal{M}[n, t]$ for some $t \leq Z$, then the assignment $\mathbf{M}^{-}$with $M_{i}^{-}:=M_{i}-\mathbf{1}_{i=u}$ minimizes $\Delta_{g}(\mathbf{p}, \cdot)$ over $\mathcal{M}[n, t-1]$, where $u:=\arg \min _{i=1, \ldots, n} \Delta^{i}[0 \rightarrow-1 ; \mathbf{M}, Z]$.

4.3.2 Theoretical Analysis: Runtime. We next establish that Algorithm 3 halts by showing the loops in Step 3 and Step 4 execute for at most $n$ iterations. Recall that Theorem 4.10 established that if the loop in Step 3 halts, then it halts with an optimal assignment. The next two theorems together establish this loop halts in at most $n$ iterations.

Theorem 4.13. In the loop in Step 3 of Algorithm 3, there is no index $j \in[n]$ for which $M_{j}$ is incremented at some iteration of the loop and then decremented at a later iteration.

Proof. The proof is by contradiction. Suppose that iteration $s$ is the first iteration of the loop where some index $j$ was decremented, having only experienced increments (if any) in the previous iterations $1,2, \ldots, s-1$. Let $r \leq s-1$ be the iteration at which $j$ was most recently incremented, and $j^{\prime \prime}$ the index of the element which was decremented at iteration $r$ so that

$$
\Delta^{j}\left[0 \rightarrow 1 ; \mathbf{M}_{r}\right]+\Delta^{j^{\prime \prime}}\left[0 \rightarrow-1 ; \mathbf{M}_{r}\right]<0,
$$

where $\mathbf{M}_{q}$ denotes the assignment at the beginning of any iteration $q(q=1, \ldots, s)$.

The following hold:

$$
\begin{aligned}
\Delta^{j^{\prime}}\left[0 \rightarrow 1 ; \mathbf{M}_{s}\right]+\Delta^{j}\left[0 \rightarrow-1 ; \mathbf{M}_{s}\right] & <0, \\
\Delta^{j}\left[0 \rightarrow 1 ; \mathbf{M}_{r}\right] & =-\Delta^{j}\left[0 \rightarrow-1 ; \mathbf{M}_{s}\right], \\
\Delta^{j^{\prime}}\left[0 \rightarrow 1 ; \mathbf{M}_{r}\right] & \leq \Delta^{j^{\prime}}\left[0 \rightarrow 1 ; \mathbf{M}_{s}\right],
\end{aligned}
$$

where (32) follows from the fact that $j$ is decremented at iteration $s$ and $j^{\prime}$ is the corresponding index which was incremented that gives a net decrease in the error; (33) follows from the hypothesis that $r$ was the most recent iteration at which $j$ was incremented; and (34) follows from the hypothesis on iteration $s$, which implies that $j^{\prime}$ must have only experienced increments at iterations $1, \ldots, s-1$ and the property of $\Delta^{j^{\prime}}$ from (18). These together yield

$$
\Delta^{j^{\prime}}\left[0 \rightarrow 1 ; \mathbf{M}_{r}\right] \leq \Delta^{j^{\prime}}\left[0 \rightarrow 1 ; \mathbf{M}_{s}\right]<-\Delta^{j}\left[0 \rightarrow 1 ; \mathbf{M}_{s}\right]=\Delta^{j}\left[0 \rightarrow 1 ; \mathbf{M}_{r}\right]
$$

where the first inequality follows from (34), the second inequality from (32), and the final equality from (33). But (35) implies that the pair of indices $\left(j, j^{\prime \prime}\right)$ selected (31) at iteration $r$ was not an optimal choice, a contradiction.

Theorem 4.14. The loop in Step 3 of Algorithm 3 halts in at most $n$ iterations. 
Proof. Theorem 4.13 establishes that once an item is decremented it will never incremented at a future step; and once an item is incremented it will never be decremented at a future step. To prove the bound of halting within $n$ iterations, we show that there are at most $n$ increments/decrements in total. We proceed by a case analysis on the generating function $g$.

Case 1: $g>0$ is a positive generator. In this case, we argue that the values $\left(M_{1}, \ldots, M_{n}\right)$ obtained in Step 1 are already initialized to the global minimum, and so the loop in Step 3 is never entered. By the hypothesis $g>0$, it follows that $g$ is decreasing on $(0,1)$ and increasing on $(1, \infty)$ :

$$
g\left(\frac{0}{Z p_{i}}\right)>\cdots>g\left(\frac{\left\lfloor Z p_{i}\right\rfloor}{Z p_{i}}\right), \quad g\left(\frac{\left\lfloor Z p_{i}\right\rfloor+1}{Z p_{i}}\right)<\cdots<g\left(\frac{Z}{Z p_{i}}\right) .
$$

Therefore, the function $g_{i}(m):=p_{i} g\left(m /\left(Z p_{i}\right)\right)$ attains its minimum at either $m=\left\lfloor Z p_{i}\right\rfloor$ or $m=$ $\left\lfloor Z p_{i}\right\rfloor+1$. Since the objective function is a linear sum of the $g_{i}$, minimizing each term individually attains the global minimum. The loop in Step 3 thus executes for zero iterations.

Case 2: $g>0$ on $(1, \infty)$ and $g<0$ on an interval $(\gamma, 1)$ for some $0<\gamma<1$. The main indices $i$ of interest are those for which

$$
\gamma<\frac{\left\lfloor Z p_{i}\right\rfloor}{Z p_{i}}<1<\frac{\left\lfloor Z p_{i}\right\rfloor+1}{Z p_{i}}
$$

since all indices for which $g\left(\left\lfloor Z p_{i}\right\rfloor /\left(Z p_{i}\right)\right)>0$ and $g\left(\left(\left\lfloor Z p_{i}\right\rfloor+1\right) /\left(Z p_{i}\right)\right)>0$ are covered by the previous case. Therefore we may assume that

$$
\gamma \leq \min _{i=1, \ldots, n}\left(\frac{\left\lfloor Z p_{i}\right\rfloor}{Z p_{i}}\right)
$$

with $g$ increasing on $(\gamma, \infty)$. (The proof for general $\gamma$ is a straightforward extension of the proof presented here.) We argue that the loop maintains the invariant $M_{i} \leq\left\lfloor Z p_{i}\right\rfloor+1$ for each $i=1, \ldots, n$.

The proof is by induction on the iterations of the loop. For the base case, observe that

$$
g\left(\frac{\left\lfloor Z p_{i}\right\rfloor}{Z p_{i}}\right)<0<g\left(\frac{\left\lfloor Z p_{i}\right\rfloor+1}{Z p_{i}}\right) \quad(i=1, \ldots, n),
$$

which follows from the hypothesis on $g$ in this case. The values after Step 1 are thus $M_{i}=\left\lfloor Z p_{i}\right\rfloor$ for each $i=1, \ldots, n$. The first iteration performs one increment/decrement so the bound holds.

For the inductive case, assume that the invariant holds for iterations $2, \ldots, s-1$. Assume, towards a contradiction, that in iteration $s$ there exists $M_{j}=\left\lfloor Z p_{j}\right\rfloor+1$ and $M_{j}$ is incremented. Let $M_{u}$ be the corresponding element that is decremented. We analyze two cases on $M_{u}$.

Subcase 2.1: $M_{u} /\left(Z p_{u}\right) \leq 1$. Then $M_{u}=\left\lfloor Z p_{u}\right\rfloor-a$ for some integer $a \geq 0$. But then

$$
\left(M_{u}-a-1\right) / Z p_{u}<\left(M_{u}-a\right) / Z p_{u}<1<\left(M_{j}+1\right) / Z p_{j}<\left(M_{j}+2\right) / Z p_{j}
$$

and

$$
\begin{aligned}
p_{j} g\left(\frac{M_{j}+2}{Z p_{j}}\right)+p_{u} g\left(\frac{M_{u}-a-1}{Z p_{u}}\right)<p_{j} g\left(\frac{M_{j}+1}{Z p_{j}}\right)+p_{u} g\left(\frac{M_{u}-a}{Z p_{u}}\right) \\
\Longleftrightarrow \frac{g\left(\frac{M_{j}+2}{Z p_{j}}\right)-g\left(\frac{M_{j}+1}{Z p_{j}}\right)}{1 /\left(Z p_{j}\right)}<\frac{g\left(\frac{M_{u}-a}{Z p_{u}}\right)-g\left(\frac{M_{u}-a-1}{Z p_{u}}\right)}{1 /\left(Z p_{u}\right)},
\end{aligned}
$$

a contradiction to the convexity of $g$.

Subcase 2.2: $1 \leq M_{u} /\left(Z p_{u}\right)$. By the inductive hypothesis, it must be that $M_{u}=\left\lfloor Z p_{u}\right\rfloor+1$. Since the net error of the increment and corresponding decrement is negative in the if branch of Step $3, \Delta^{j}[1 \rightarrow 2]+\Delta^{l}[1 \rightarrow 0]<0$, which implies

$$
\Delta^{j}[1 \rightarrow 2]<-\Delta^{l}[1 \rightarrow 0]=\Delta^{l}[0 \rightarrow 1] .
$$


Since $\Delta^{j}[0 \rightarrow 1]<\Delta^{j}[1 \rightarrow 2]$ from (18), it follows that $M_{j}$ should have been incremented at two previous iterations before having incremented $M_{u} \leftarrow M_{u}+1$, contradicting the minimality of the increments at each iteration.

Since each $M_{i}$ is one greater than the initial value at the termination of the loop, and at each iteration one value is incremented, the loop terminates in at most $n$ iterations.

Case 3: $g>0$ on $(0,1)$ and $g<0$ on some interval $(1, \gamma)$ for $1<\gamma \leq \infty$. The proof is symmetric to the previous case, with initial values $M_{i}=\left\lfloor Z p_{i}\right\rfloor+1$ from Step 1 and invariant $M_{i} \geq\left\lfloor Z p_{i}\right\rfloor$.

Remark 4.15. The overall cost of Step 3 is $O(n \log n)$, since $\left(j, j^{\prime}\right)$ can be found in $O(\log n)$ time by performing order-preserving insertions and deletions on a pair of initially sorted lists.

Theorem 4.16. The value $S$ defined in Step 4 of Algorithm 3 always satisfies $-(n-1) \leq S \leq n-1$.

Proof. The smallest value of $S$ is obtained when each $M_{i}=\left\lfloor Z p_{i}\right\rfloor$, in which case

$$
0 \leq \sum_{i=1}^{n}\left(Z p_{i}-\left\lfloor Z p_{i}\right\rfloor\right):=\sum_{i=1}^{n} \chi\left(Z p_{i}\right) \leq n-1,
$$

where the first inequality follows from $\lfloor x\rfloor \leq x$ and the final inequality from the fact that $0 \leq$ $\chi(x)<1$ so that the integer $\sum_{i=1}^{n} \chi\left(Z p_{i}\right)<n$. Therefore, $-S \leq(n-1) \Longrightarrow-(n-1) \leq S$. Similarly, the largest value of $S$ is obtained when each $M_{i}=\left\lfloor Z p_{i}\right\rfloor+1$, so that

$$
\sum_{i=1}^{n}\left(\left\lfloor Z p_{i}\right\rfloor+1-Z p_{i}\right)=\sum_{i=1}^{n}\left(1-\chi\left(Z p_{i}\right)\right)=n-\sum_{i=1}^{n} \chi\left(Z p_{i}\right) \leq n-1 .
$$

Therefore, $S \leq n-1$, where the final inequality uses the fact that $\chi\left(Z p_{i}\right) \neq 0$ for some $i$ (otherwise, $M_{i}=\left\lfloor Z p_{i}\right\rfloor$ would be the optimum for each $\left.i\right)$.

Theorems 4.10-4.16 together imply Theorem 4.7. Furthermore, using the implementation given in Remark 4.15, the overall runtime of Algorithm 3 is order $n \log n$.

Returning to Problem 2.15, from Theorems 3.4 and Theorem 4.7, the approximation error can be minimized over the set of output distributions of all entropy-optimal $k$-bit samplers as follows: (i) for each $j=0, \ldots, k$, let $\mathbf{M}_{j}$ be the optimal $Z_{k j}$-type distribution for approximating $\mathbf{p}$ returned by Algorithm 3; (ii) let $l=\arg \min _{0 \leq j \leq k} \Delta_{g}\left(\mathbf{p}, \mathbf{M}_{j}\right)$; (iii) set $\hat{p}_{i}:=M_{l i} / Z_{k l}(i=1, \ldots, n)$. The optimal probabilities for any sampler that halts after consuming at most $k$ bits (as in Proposition 2.16) are given by $\hat{p}_{i}:=M_{k i} / Z_{k k}$. The next theorem establishes that, when the $f$-divergence is total variation, the approximation error decreases proportionally to $1 / Z$ (the proof is in the appendix).

THEOREM 4.17. If $\Delta_{g}$ is the total variation divergence, then any optimal solution $\mathbf{M}$ returned by Algorithm 3 satisfies $\Delta_{g}(\mathbf{p}, \mathbf{M}) \leq n / 2 Z$.

\section{CONSTRUCTING ENTROPY-OPTIMAL SAMPLERS}

Now that we have described how to find a closest-approximation distribution for Problem 4.6 using Algorithm 3, we next describe how to efficiently construct an entropy-optimal sampler.

Suppose momentarily that we use the rejection method (Algorithm 1) described in Section 1.1, which can sample exactly from any $Z$-type distribution, which includes all distributions returned by Algorithm 3. Since any closest-approximation distribution that is the output distribution of a $k$-bit entropy-optimal sampler has denominator $Z=Z_{k l} \leq 2^{k}$, rejection sampling needs exactly $k$ bits of precision. The expected number of trials is $2^{k} / Z$ and $k$ random bits are used per trial, so that $k 2^{k} / Z \leq 2 k$ bits per sample are consumed on average. The following example illustrates that the entropy consumption of the rejection method can be exponentially larger than the entropy of $\mathbf{p}$. 
Example 5.1. Let $\mathbf{p}=\left(a_{1} / 2^{k}, \ldots, a_{n} / 2^{k}\right)$ with $n=k$. An entropy-optimal sampler uses at most $\log n$ bits per sample (Theorem 2.9), whereas rejection (Algorithm 1) uses $n$ bits per sample.

We thus turn our attention toward constructing an entropy-optimal sampler that realizes the entropy-optimality guarantees from Theorem 2.9. For the data structures in this section we use a zero-based indexing system. For positive integers $l$ and $k$, let $\mathbf{M}:=\left(M_{0}, \ldots, M_{n-1}\right)$ be the return value of Algorithm 3 given denominator $Z_{k l}$. Without loss of generality, we assume that (i) $k, l$, and $M_{i}$ have been reduced so that some probability $M_{i} / Z_{k l}$ is in lowest terms; and (ii) for each $j$ we have $M_{j}<Z_{k l}$ (if $M_{j}=Z_{k l}$ for some $j$, then the sampler is degenerate: it always returns $j$ ).

Algorithm 4 shows the first stage of the construction, which returns the binary probability matrix $\mathbf{P}$ of $\mathbf{M}$. The $i$ th row contains the first $k$ bits in the concise binary expansion of $M_{i} / Z_{k l}$, where first $l$ columns encode the finite prefix and the final $k-l$ columns encode the infinitely repeating suffix Algorithm 5 shows the second stage, which converts $\mathbf{P}$ from Algorithm 4 into an entropy-optimal DDG tree $T$. From Theorem 2.9, $T$ has a node labeled $r$ at level $c+1$ if and only if $\mathrm{P}[r, c]=1$ (recall the root is at level 0 , so column $c$ of $\mathbf{P}$ corresponds to level $c+1$ of $T$ ). The MakeLeafTABLe function returns a hash table $L$ that maps the level-order integer index $i$ of any leaf node in a complete binary tree to its label $L[i] \in\{1, \ldots, n\}$ (the index of the root is zero). The labeling ensures that leaf nodes are filled right-to-left and are labeled in increasing order. Next, we define a node data structure with fields left, right, and label, indicating the left child, right child, and outcome label (for leaf nodes). The MAKETREe function builds the tree $T$ from $L$, returning the root node. The function stores the list $A$ of ancestors at level $l$ in right-to-left order, and constructs back-edges from any non-leaf node at level $k-1$ to the next available ancestor at level $l$ to encode the recurring subtree.

Algorithm 6 shows the third stage, which converts the root node of the entropy-optimal DDG tree $T$ returned from Algorithm 5 into a sampling-efficient encoding enc. The PACKTrEe function fills the array enc such that for an internal node $i$, enc $[i]$ and enc $[i+1]$ store the indexes of enc for the left and right child (respectively) if $i$ is a branch; and for an leaf node $i$, enc $[i]$ stores the label (as a negative integer). The field node.loc tracks back-edges, pointing to the ancestor instead of making a recursive call whenever a node has been visited by a previous recursive call.

Now that preprocessing is complete, Algorithm 7 shows the main sampler, which uses the enc data structure from Algorithm 6 and the flip() primitive to traverse the DDG tree starting from the root (at enc[0]). Since PACKTREe uses negative integers to encode the labels of leaf nodes, the SAMPLEENCODING function returns the outcome $-e n c[c]$ whenever enc $[c]<0$. Otherwise, the sampler goes to the left child (at enc[c]) if flip() returns 0 or the right child (at enc[c+1]) if flip() returns 1 . The resulting sampler is very efficient and only stores the linear array enc in memory, whose size is order $n k$. (The DDG tree of an entropy-optimal $k$-bit sampler is a complete depth- $k$ binary tree with at most $n$ nodes per level, so there are at most $n k$ leaf nodes and $n k$ branch nodes.)

For completeness, we also present Algorithm 8, which implements an entropy-optimal sampler by operating directly on the $n \times k$ matrix $\mathbf{P}$ returned from Algorithm 4 . This algorithm is based on a recursive extension of the Knuth and Yao sampler given in Roy et al. [2013], where we allow for an infinitely repeating suffix by resetting the column counter $c$ to $l$ whenever $c=k-1$ is at the last columns. (The algorithm in Roy et al. [2013] is designed for hardware and samples from a probability matrix with a finite number of columns and no repeating suffixes. Unlike the focus of this paper, Roy et al. [2013] does not deliver closest-approximation distributions for limited-precision sampling.) Algorithm 7 is significantly more efficient as its runtime is dictated by the entropy (upper bounded by $\log n$ ) whereas the runtime of Algorithm 8 is upper bounded by $n \log n$ due to the order $n$ inner loop. Figure 4 in Section 6.2.3 shows that, when implemented in software, the increase in algorithmic efficiency from using a dense encoding can deliver wall-clock gains of up to $5000 \mathrm{x}$ on a representative sampling problem. 

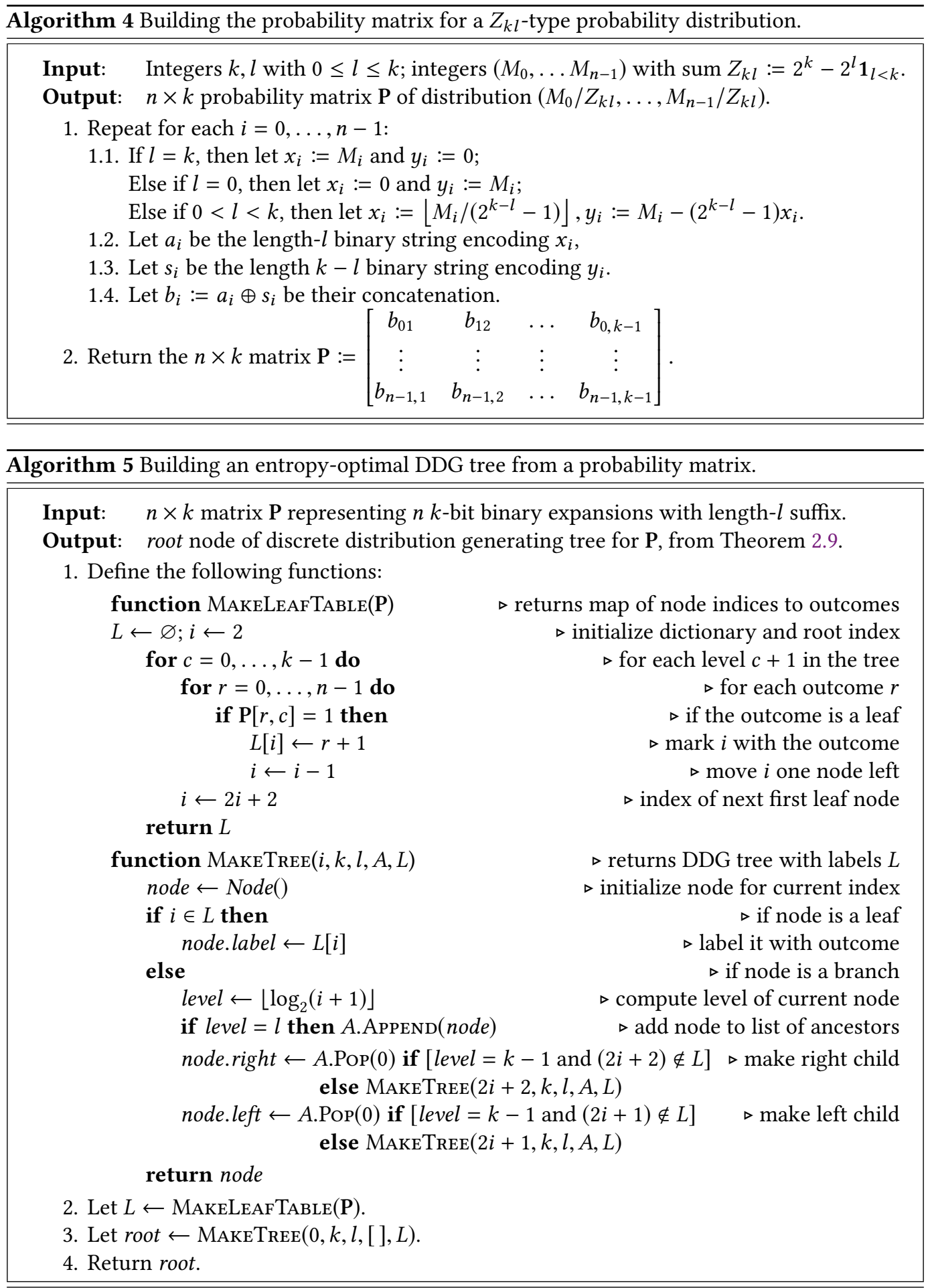

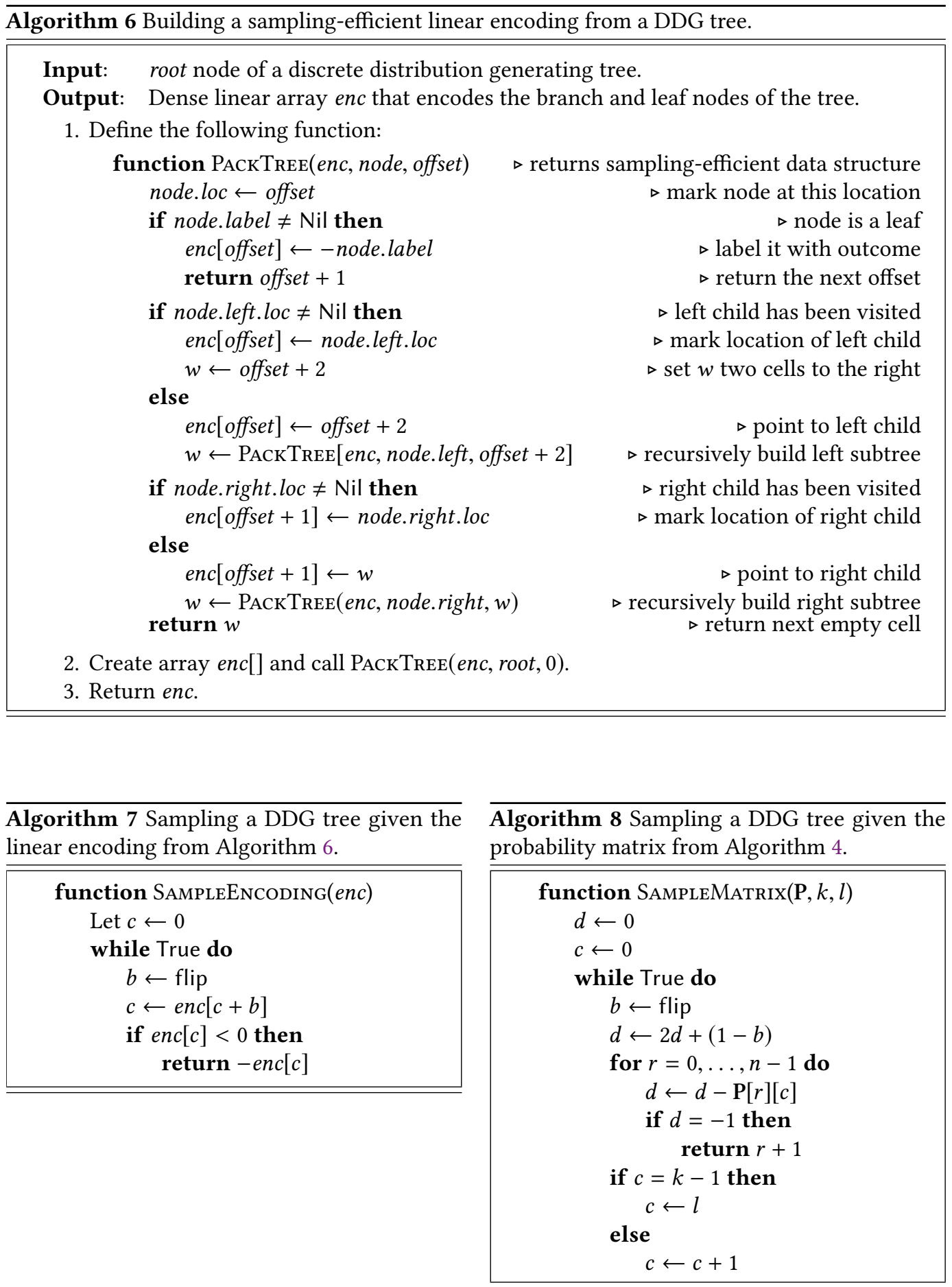


\section{EXPERIMENTAL RESULTS}

We next evaluate the optimal limited-precision sampling algorithms presented in this paper. Section 6.1 investigates how the error and entropy consumption of the optimal samplers vary with the parameters of common families of discrete probability distributions. Section 6.2 compares the optimal samplers with two limited-precision baselines samplers, showing that our algorithms are up to $1000 \mathrm{x}-10000 \mathrm{x}$ more accurate, consume up to $10 \mathrm{x}$ fewer random bits per sample, and are $10 \mathrm{x}-100 \mathrm{x}$ faster in terms of wall-clock time. Section 6.3 compares our optimal samplers to exact samplers on a representative binomial distribution, showing that exact samplers can require high precision or consume excessive entropy, whereas our optimal approximate samplers can use less precision and/or entropy at the expense of a small sampling error. The appendix contains a study of how the closest-approximation error varies with the precision specification and entropy of the target distribution, as measured by three different $f$-divergences. The online artifact contains the experiment code. All $\mathrm{C}$ algorithms used for measuring performance were compiled with gcc level 3 optimizations, using Ubuntu 16.04 on AMD Opteron $63761.4 \mathrm{GHz}$ processors.

\subsection{Characterizing Error and Entropy for Families of Discrete Distributions}

We study how the approximation error and entropy consumption of our optimal approximate samplers vary with the parameter values of four families of probability distributions: (i) $\operatorname{Binomial}(n, p)$ : the number of heads in $n$ independent tosses of a biased $p$-coin; (ii) $\operatorname{Beta} \operatorname{Binomial}(n, \alpha, \beta)$ : the number of heads in $n$ independent tosses of a biased $p$-coin, where $p$ is itself randomly drawn from a $\operatorname{Beta}(\alpha, \beta)$ distribution; (iii) Discrete $\operatorname{Gaussian}(n, \sigma)$ : a discrete Gaussian over the integers $\{-n, \ldots, n\}$ with variance $\sigma^{2}$; and (iv) Hypergeometric $(n, m, d)$ : the number of red balls obtained after $d$ draws (without replacement) from a bin that has $m$ red balls and $n-m$ blue balls.

Figure 2 shows how the closest-approximation error (top row) and entropy consumption (bottom row) vary with two of the parameters of each family ( $\mathrm{x}$ and y-axes) when using $k=32$ bits of precision. Since Beta Binomial and Hypergeometric have three parameters, we fix $n=80$ and vary the remaining two parameters. Closest-approximation distributions are obtained from Algorithm 3, using $Z=2^{32}$ and the Hellinger divergence (which is most sensitive at medium entropies). The plots show that, even with the same family, the closest-approximation error is highly dependent on the target distribution and the interaction between parameter values. For example, in Figure $2 \mathrm{a}$
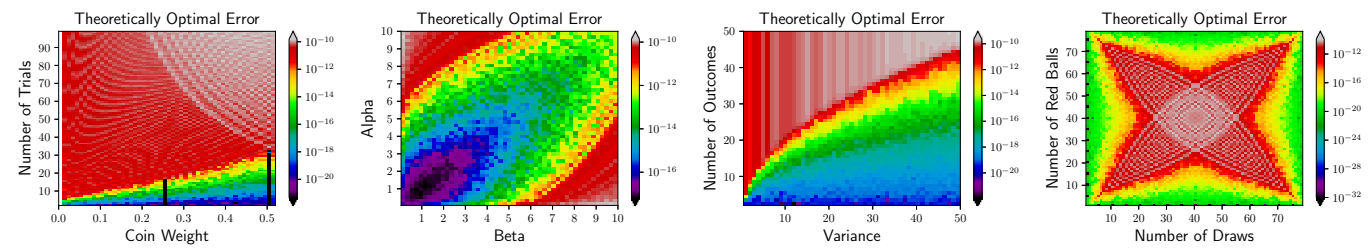

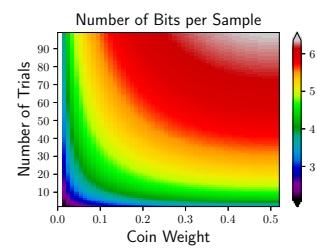

(a) Binomial

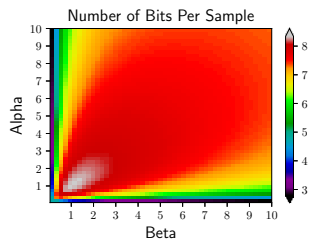

(b) Beta Binomial $(n=80)$

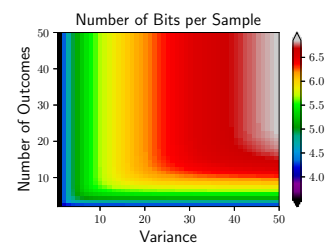

(c) Discrete Gaussian

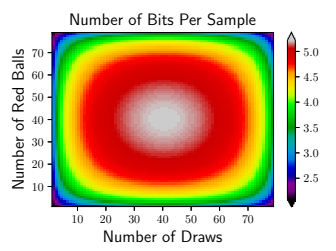

(d) Hypergeometric $(n=80)$

Fig. 2. Characterization of the theoretically optimal approximation error (top row) and average number of bits per sample (bottom row) for four common families of probability distributions using $k=32$ bits of precision. 
(top panel), the black spikes at coin weight 0.25 and 0.50 correspond to pairs $(n, p)$ where the binomial distribution can be sampled exactly. Moreover, for a fixed coin weight ( $\mathrm{x}$-axis), the error increases as the number of trials (y-axis) increases. The rate at which the error increases with the number of trials is inversely proportional to the coin weight, which is mirrored by the fact that the average number of bits per sample (bottom panel) varies over a wider range and at a faster rate at low coin weights than at high coin weights. In Figure 2c, for a fixed level of variance (x-axis), the error increases until the number of outcomes (y-axis) exceeds the variance, after which the tail probabilities become negligible. In Figure $2 \mathrm{~d}$ when the number of red balls $m$ and number of draws $d$ are equal to roughly half of the population size $n$, the bits per sample and approximation error are highest (grey in center of both panels). This relationship stands in contrast to Figure 2b, where approximation error is lowest (black/purple in lower left of top panel) when bits per sample is highest (grey in lower left of bottom panel). The methods presented in this paper enable rigorous and systematic assessments of the effects of bit precision on theoretically-optimal entropy consumption and sampling error, as opposed to empirical, simulation-based assessments of entropy and error which can be very noisy in practice (e.g., Jonas [2014, Figure 3.15]).

\subsection{Comparing Error, Entropy, and Runtime to Baseline Limited-Precision Algorithms}

We next show that the proposed sampling algorithm is more accurate, more entropy-efficient, and faster than existing limited-precision sampling algorithms. We briefly review two baselines below.

Inversion sampling. Recall from Section 1.1 that inversion sampling is a universal method based on the key property in Eq. (1). In the $k$-bit limited-precision setting, a floating-point number $U^{\prime}$ (with denominator $2^{k}$ ) is used to approximate a real uniform variate $U$. The GNU $\mathrm{C}++$ standard library [Lea 1992] v5.4.0 implements inversion sampling as in Algorithm 2 (using $\leq$ instead of $<)^{3}$ As $W \sim$ Uniform $\left(\left\{0,1 / 2^{k}, \ldots,\left(2^{k}-1\right) / 2^{k}\right\}\right)$, it can be shown that the limited-precision inversion sampler has the following output probabilities $\hat{p}_{i}$, where $\widetilde{p}_{j}:=\sum_{s=1}^{j} p_{s}(j=1, \ldots, n)$ and $2 \leq i \leq n$ :

$$
\hat{p}_{1} \propto\left\lfloor 2^{k} \widetilde{p}_{1}\right\rfloor+1_{\widetilde{p}_{1} \neq 1} ; \quad \hat{p}_{i} \propto\left\{\begin{array}{ll}
\max \left(0,\left\lceil 2^{k} \widetilde{p}_{i}\right\rceil-\left\lfloor 2^{k} \widetilde{p}_{i-1}\right\rfloor\right) & \left(\text { if } 2^{k} \widetilde{p}_{i}=\left\lfloor 2^{k} \widetilde{p}_{i}\right\rfloor \text { and } \widetilde{p}_{i} \neq 1\right) \\
\max \left(0,\left\lceil 2^{k} \widetilde{p}_{i}\right\rceil-\left\lfloor 2^{k} \widetilde{p}_{i-1}\right\rfloor-1\right) & \text { (otherwise) }
\end{array} .\right.
$$

Interval algorithm [Han and Hoshi 1997]. This method implements inversion sampling by recursively partitioning the unit interval $[0,1]$ and using the cumulative distribution of $\mathbf{p}$ to lazily find the bin in which a uniform random variable falls. We refer to Uyematsu and Li [2003, Algorithm 1] for a limited-precision implementation of the interval algorithm using $k$-bit integer arithmetic.

6.2.1 Error Comparison. Both the inversion and interval samplers use at most $k$ bits of precision, which, from Proposition 2.16, means that these algorithms are less accurate than the optimal approximate samplers from Algorithm 3 (using $Z=2^{k}$ ) and less entropy-efficient than the sampler in Algorithm 7. To compare the errors, 500 distributions are obtained by sweeping through a grid of values that parameterize the shape and dimension for each of six families of probability distributions. For each target distribution, probabilities from the inversion method (from Eq. (46)), the interval method (computed by enumeration), and the optimal approximation (from Algorithm 3) are obtained using $k=16$ bits of precision. In Figure 3, the $\mathrm{x}$-axis shows the approximation error (using the Hellinger divergence) of each method relative to the theoretically-optimal error achieved by our samplers. The y-axis shows the fraction of the 500 distributions whose relative error is less than or equal to the value on the $\mathrm{x}$-axis. The results show that, for this benchmark set, the output distributions of inversion and interval samplers are up to three orders of magnitude less accurate relative to the output distribution of the optimal $k$-bit approximation delivered by our algorithm.

\footnotetext{
${ }^{3}$ Steps 1 and 2 are implemented in generate_canonical and Step 3 is implemented in discrete_distribution: : operator() using a linear scan; see /gcc-5.4.0/libstdc++v3/include/bits/random. tcc in https://ftp.gnu.org/gnu/gcc/gcc-5.4.0/gcc-5.4.0.tar.gz.
} 
Table 2. Comparison of the average number of input bits per sample used by inversion sampling, interval sampling, and the proposed method, in each of the six parameterized families using $k=16$ bits of precision.

\begin{tabular}{lrrr}
\hline \multirow{2}{*}{ Distribution } & \multicolumn{3}{c}{ Average Number of Bits per Sample } \\
\cline { 2 - 4 } & Inversion Sampler (Alg. 2) & Interval Sampler [Uyematsu and Li 2003] & Optimal Sampler (Alg. 7) \\
\hline Benford & 16 & 6.34 & 5.71 \\
Beta Binomial & 16 & 4.71 & 4.16 \\
Binomial & 16 & 5.05 & 4.31 \\
Boltzmann & 16 & 1.51 & 1.03 \\
Discrete Gaussian & 16 & 6.00 & 5.14 \\
Hypergeometric & 16 & 4.04 & 3.39 \\
\hline
\end{tabular}
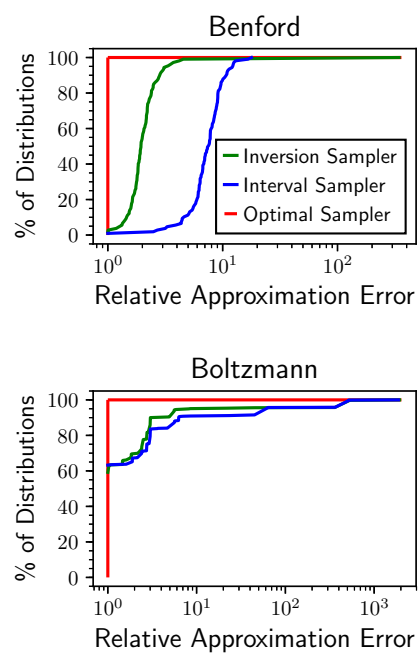
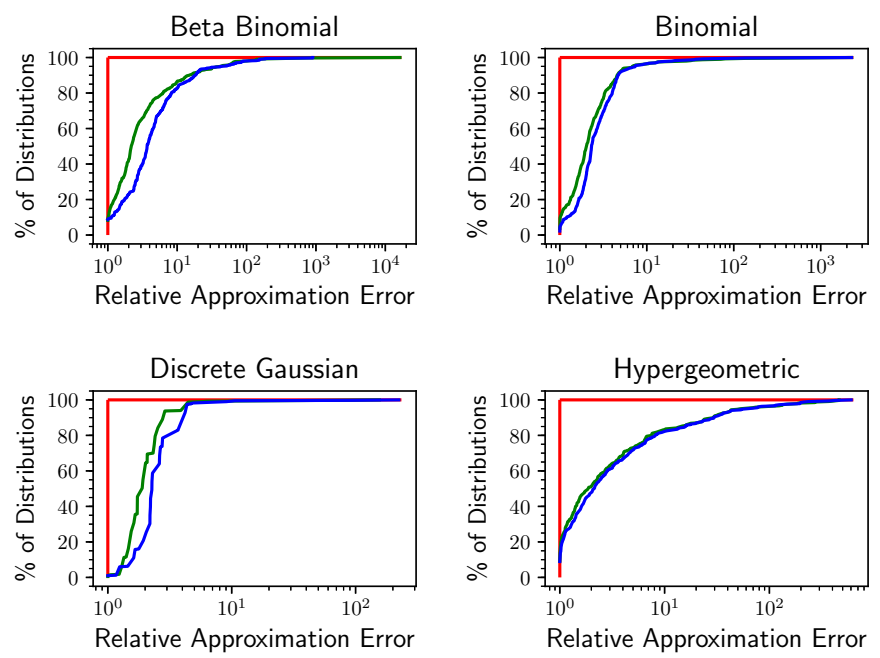

Fig. 3. Comparison of the approximation error of limited-precision implementations of interval sampling (green) and inversion sampling (blue) relative to error obtained by the optimal sampler (red), for six families of probability distributions using $k=16$ of bits precision. The $\mathrm{x}$-axis shows the approximation error of each sampler relative to the optimal error. The $y$-axis shows the fraction of 500 distributions from each family whose relative error is less than or equal to the corresponding value on the $\mathrm{x}$-axis.

6.2.2 Entropy Comparison. Next, we compare the efficiency of each sampler measured in terms of the average number of random bits drawn from the source to produce a sample, shown in Table 2. Since these algorithms are guaranteed to halt after consuming at most $k$ random bits, the average number of bits per sample is computed by enumerating over all $2^{k}$ possible $k$-bit strings (using $k=16$ gives 65536 possible input sequences from the random source) and recording, for each sequence of input bits, the number of consumed bits until the sampler halts. The inversion algorithm consumes all $k$ available bits of entropy, unlike the interval and optimal samplers, which lazily draw bits from the random source until an outcome can be determined. For all distributional families, the optimal sampler uses fewer bits per sample than are used by interval sampling.

6.2.3 Runtime Comparison. We next assess the runtime performance of our sampling algorithms as the dimension and entropy of the target distribution increases. For each $n \in\{10,100,1000,10000\}$, we generate 1000 distributions with entropies ranging from $0, \ldots, \log (n)$. For each distribution, we measure the time taken to generate a sample based on 100000 simulations according to four methods: the optimal sampler using SAMPLEENCODING (Algorithm 7); the optimal sampler using 

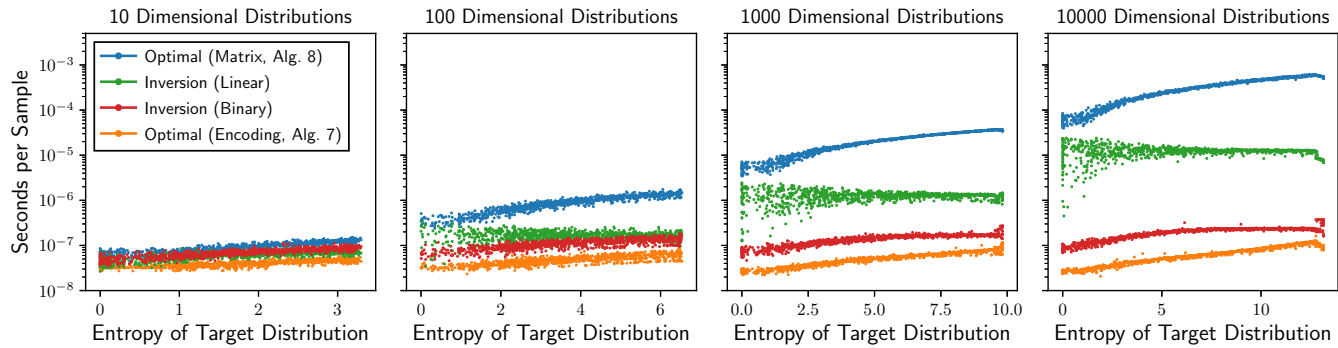

Fig. 4. Comparison of wall-clock time per sample and order of growth of two implementations of the optimal samplers (using Algorithms 7 and 8) with inversion sampling (using linear and binary search in Algorithm 2).

Table 3. Comparison of runtime and number of calls to the random number generator using limited-precision entropy-optimal and inversion sampling to generate 100 million samples from 100 dimensional distributions

\begin{tabular}{lrrr}
\hline Method & Entropy of Target Distribution & Number of PRNG Calls & PRNG Wall-Clock Time (ms) \\
\hline & 0.5 & $7,637,155$ & 120 \\
Optimal Approximate & 2.5 & $11,373,471$ & 160 \\
Sampler (Alg. 7) & 4.5 & $18,879,900$ & 260 \\
& 6.5 & $24,741,348$ & 350 \\
\hline Inversion Sampler (Alg. 2) & (all) & $100,000,000$ & 1410 \\
\hline
\end{tabular}

SAMPleMATrix (Algorithm 8); the inversion sampler using a linear scan (Algorithm 2, as in the GNU C++ standard library); and the inversion sampler using binary search (fast C implementation). Figure 4 shows the results, where the $\mathrm{x}$-axis is the entropy of the target distribution and the y-axis is seconds per sample (log scale). In general, the difference between the samplers increases with the dimension $n$ of the target distribution. For $n=10$, the SAMPLEENCODING sampler executes a median of over 1.5x faster than any other sampler. For $n=10000$, SAMPLEENCODING executes a median of over 3.4x faster than inversion sampling with binary search and over 195x faster than the linear inversion sampler implemented in the C++ library. In comparison with SAMPLEMATRIX [Roy et al. 2013], SAMPLeENCODing is faster by a median of $2.3 \mathrm{x}(n=10)$ to over $5000 \mathrm{x}(n=10000)$.

The worst runtime scaling is given by SAMPLEMATRIX which, although entropy-optimal, grows order $n H(\mathbf{p})$ due to the inner loop through the rows of the probability matrix. In contrast, SAMPLEENCODING uses the dense linear array described in Section 5 and is asymptotically more efficient: its runtime depends only on the entropy $H(\mathbf{p}) \leq \log n$. As for the inversion methods, there is a significant gap between the runtime of SAMPLEENCODING (orange) and the binary inversion sampler (red) at low values of entropy, which is especially visible at $n=1000$ and $n=10000$. The binary inversion sampler scales order $\log n$ independently of the entropy, and is thus less performant than SAMPLEENCODING when $H(\mathbf{p}) \ll \log n$ (the gap narrows as $H(\mathbf{p})$ approaches $\log n$ ).

Table 3 shows the wall-clock improvements from using Algorithm 7. Floating-point sampling algorithms implemented in standard software libraries typically make one call to the pseudorandom number generator per sample, consuming a full 32-bit or 64-bit pseudorandom word, which in general is highly wasteful. (As a conceptual example, sampling Bernoulli(1/2) requires sampling only one random bit, but comparing an approximately-uniform floating-point number $U^{\prime}<0.5$ as in inversion sampling uses e.g., 64 bits.) In contrast, the optimal approximate sampler (Algorithm 7) is designed to lazily consume random bits (following Lumbroso [2013], our implementation of flip stores a buffer of pseudorandom bits equal to the word size of the machine) which results in fewer function calls to the underlying pseudorandom number generator and $4 \mathrm{x}-12 \mathrm{x}$ less wall-clock time. 
Table 4. Precision, entropy consumption, and sampling error of Knuth and Yao sampling, rejection sampling, and optimal approximate sampling, at various levels of precision for the Binomial(50,61/500) distribution.

\begin{tabular}{lrrr}
\hline Method & Precision $k_{(l)}$ & Bits per Sample & Error $\left(L_{1}\right)$ \\
\hline Exact Knuth and Yao Sampler (Thm. 2.9) & $5.6 \times 10^{104}(100)$ & 5.24 & 0.0 \\
Exact Rejection Sampler (Alg. 1) & $449_{(448)}$ & 735 & 0.0 \\
\hline & $4_{(4)}$ & 5.03 & $2.03 \times 10^{-1}$ \\
Optimal Approximate & $8_{(4)}$ & 5.22 & $1.59 \times 10^{-2}$ \\
Sampler (Alg. 3+7) & $16_{(0)}$ & 5.24 & $6.33 \times 10^{-5}$ \\
& $32_{(12)}$ & 5.24 & $1.21 \times 10^{-9}$ \\
& $64_{(29)}$ & 5.24 & $6.47 \times 10^{-19}$ \\
\hline
\end{tabular}

\subsection{Comparing Precision, Entropy, and Error to Exact Sampling Algorithms}

Recall that two algorithms for sampling from $Z$-type distributions (Definition 4.3) are: (i) exact Knuth and Yao sampling (Theorem 2.9), which samples from any $Z$-type distribution using at most $H(\mathbf{p})+2$ bits per sample and precision $k$ described in Theorem 3.4; and (ii) rejection sampling (Algorithm 1), which samples from any $Z$-type distribution using $k$ bits of precision (where $\left.2^{k-1}<Z \leq 2^{k}\right)$ using $k 2^{k} / Z$ bits per sample. Consider the Binomial(50,61/500) distribution $\mathbf{p}$ which is the number of heads in 50 tosses of a biased coin whose probability of heads is $61 / 500$. The probabilities are $p_{i}:=\left(\begin{array}{c}50 \\ i\end{array}\right)(61 / 500)^{i}(39 / 500)^{n-i}(i=0, \ldots, n)$ and $\mathbf{p}$ is a $Z$-type distribution with $Z=8.8817841970012523233890533447265625 \times 10^{134}$. Table 4 shows a comparison of the two exact samplers to our optimal approximate samplers. The first column shows the precision $k_{(l)}$, which indicates $k$ bits are used and $l$ (where $0 \leq l \leq k$ ) is the length of the repeating suffix in the number system $\mathbb{B}_{k l}$ (Section 3). Recall that exact samplers use finite but arbitrarily high precision. The second and third columns show bits per sample and sampling error, respectively.

Exact Knuth and Yao sampler. This method requires a tremendous amount of precision to generate an exact sample (following Theorem 3.5), as dictated by the large value of $Z$ for the Binomial $(50,61 / 500)$ distribution. The required precision far exceeds the amount of memory available on modern machines. Although at most 5.24 bits per sample are needed on average (two more than the 3.24 bits of entropy in the target distribution), the DDG tree has more than $10^{104}$ levels. Assuming that each level is a byte, storing the sampler would require around $10^{91}$ terabytes.

Exact rejection sampler. This method requires 449 bits of precision (roughly 56 bytes), which is the number of bits needed to encode common denominator $Z$. This substantial reduction in precision as compared to the Knuth and Yao sampler comes at the cost of higher number of bits per sample, which is roughly $150 \mathrm{x}$ higher than the information-theoretically optimal rate. The higher number of expected bits per sample leads to wasted computation and higher runtime in practice due to excessive calls to the random number generator (as illustrated in Table 3).

Optimal approximate sampler. For precision levels ranging from $k=4$ to 64 , the selected value of $l$ delivers the smallest approximation error across executions of Algorithm 3 on inputs $Z_{k k}, \ldots, Z_{k 0}$. At each precision, the number of bits per sample has an upper bound that is very close to the upper bound of the optimal rate, since the entropies of the closest-approximation distributions are very close to the entropy of the target distribution, even at low precision. Under the $L_{1}$ metric, the approximation error decreases exponentially quickly with the increase in precision (Theorem 4.17).

These results illustrate that exact Knuth and Yao sampling can be infeasible in practice, whereas rejection sampling requires less precision (though higher than what is typically available on low precision sampling devices [Mansinghka and Jonas 2014]) but is wasteful in terms of bits per sample. The optimal approximate samplers are practical to implement and use significantly less precision or bits per sample than exact samplers, at the expense of a small approximation error that can be controlled based on the accuracy and entropy constraints of the application at hand. 


\section{CONCLUSION}

This paper has presented a new class of algorithms for optimal approximate sampling from discrete probability distributions. The samplers minimize both statistical error and entropy consumption among the class of all entropy-optimal samplers and bounded-entropy samplers that operate within the given precision constraints. Our samplers lead to improvements in accuracy, entropy-efficiency, and wall-clock runtime as compared to existing limited-precision samplers, and can use significantly fewer computational resources than are needed by exact samplers.

Many existing programming languages and systems include libraries and constructs for random sampling [Lea 1992; MathWorks 1993; R Core Team 2014; Galassi et al. 2019]. In addition to the areas of scientific computing mentioned in Section 1, relatively new and prominent directions in the field of computing that leverage random sampling include probabilistic programming languages and systems [Gordon et al. 2014; Saad and Mansinghka 2016; Staton et al. 2016; Cusumano-Towner et al. 2019]; probabilistic program synthesis [Nori et al. 2015; Saad et al. 2019]; and probabilistic hardware [de Schryver et al. 2012; Dwarakanath and Galbraith 2014; Mansinghka and Jonas 2014] In all these settings, the efficiency and accuracy of random sampling procedures play a key role in many implementation techniques. As uncertainty continues to play an increasingly prominent role in a range of computations and as programming languages move towards more support for random sampling as one way of dealing with this uncertainty, trade-offs between entropy consumption, sampling accuracy, numerical precision, and wall-clock runtime will form an important set of design considerations for sampling procedures. Due to their theoretical optimality properties, ease-of-implementation, and applicability to a broad set of statistical error measures, the algorithms in this paper are a step toward a systematic and practical approach for navigating these trade-offs.

\section{ACKNOWLEDGMENTS}

This research was supported by a philanthropic gift from the Aphorism Foundation.

\section{REFERENCES}

Julia Abrahams. 1996. Generation of Discrete Distributions from Biased Coins. IEEE Trans. Inf. Theory 42, 5 (Sept. 1996), 1541-1546.

S. M. Ali and S. D. Silvey. 1966. A General Class of Coefficients of Divergence of One Distribution from Another. J. R. Stat. Soc. B. 28, 1 (Jan. 1966), 131-142.

Ziv Bar-Yossef, Thathachar S. Jayram, Ravi Kumar, and D. Sivakumar. 2004. An Information Statistics Approach to Data Stream and Communication Complexity. f. Comput. Syst. Sci. 68, 4 (June 2004), 702-732.

Kurt Binder (Ed.). 1986. Monte Carlo Methods in Statistical Physics (2 ed.). Topics in Current Physics, Vol. 7. Springer-Verlag, Berlin.

Antonio Blanca and Milena Mihail. 2012. Efficient Generation $\epsilon$-close to $G(n, p)$ and Generalizations. (April 2012). arXiv:1204.5834

Lenore Blum, Felipe Cucker, Michael Shub, and Steve Smale. 1998. Complexity and Real Computation. Springer-Verlag, New York.

Manuel Blum. 1986. Independent Unbiased Coin Flips from a Correlated Biased Source: A Finite State Markov Chain. Combinatorica 6, 2 (June 1986), 97-108.

Karl Bringmann and Tobias Friedrich. 2013. Exact and Efficient Generation of Geometric Random Variates and Random Graphs. In ICALP 2013: Proceedings of the 40th International Colloquium on Automata, Languages and Programming (Riga, Latvia). Lecture Notes in Computer Science, Vol. 7965. Springer, Heidelberg, 267-278.

Karl Bringmann and Konstantinos Panagiotou. 2017. Efficient Sampling Methods for Discrete Distributions. Algorithmica 79, 2 (Oct. 2017), 484-508.

Ferdinando Cicalese, Luisa Gargano, and Ugo Vaccaro. 2006. A Note on Approximation of Uniform Distributions from Variable-to-Fixed Length Codes. IEEE Trans. Inf. Theory 52, 8 (Aug. 2006), 3772-3777.

Thomas M. Cover and Joy A. Thomas. 2006. Elements of Information Theory (2 ed.). John Wiley \& Sons, Inc., Hoboken.

Marco F. Cusumano-Towner, Feras A. Saad, Alexander K. Lew, and Vikash K. Mansinghka. 2019. Gen: A General-purpose Probabilistic Programming System with Programmable Inference. In PLDI 2019: Proceedings of the 40th ACM SIGPLAN 
Conference on Programming Language Design and Implementation (Phoenix, AZ, USA). ACM, New York, 221-236.

Christian de Schryver, Daniel Schmidt, Norbert Wehn, Elke Korn, Henning Marxen, Anton Kostiuk, and Ralf Korn. 2012. A Hardware Efficient Random Number Generator for Nonuniform Distributions with Arbitrary Precision. Int. f. Reconf. Comput. 2012, Article 675130 (2012), 11 pages.

Luc Devroye. 1982. A Note on Approximations in Random Variate Generation. J. Stat. Comput. Simul. 14, 2 (1982), $149-158$. Luc Devroye. 1986. Non-Uniform Random Variate Generation. Springer-Verlag, New York.

Luc Devroye and Claude Gravel. 2015. Sampling with Arbitrary Precision. (Feb. 2015). arXiv:1502.02539

Inderjit S. Dhillon, Subramanyam Mallela, and Rahul Kumar. 2003. A Divisive Information-Theoretic Feature Clustering Algorithm for Text Classification. F. Mach. Learn. Res. 3 (March 2003), 1265-1287.

Dragan Djuric. 2019. Billions of Random Numbers in a Blink of an Eye. Retrieved June 15, 2019 from https://dragan.rocks/ articles/19/Billion-random-numbers-blink-eye-Clojure

Chaohui Du and Guoqiang Bai. 2015. Towards Efficient Discrete Gaussian Sampling For Lattice-Based Cryptography. In FPL 2015: Proceedings of the 25th International Conference on Field Programmable Logic and Applications (London, UK). IEEE Press, Piscataway, 1-6.

Nagarjun C. Dwarakanath and Steven D. Galbraith. 2014. Sampling from Discrete Gaussians for Lattice-Based Cryptography On a Constrained Device. Appl. Algebr. Eng. Comm. 25, 3 (June 2014), 159-180.

Peter Elias. 1972. The Efficient Construction of an Unbiased Random Sequence. Ann. Math. Stat. 43, 3 (June 1972), 865-870.

János Folláth. 2014. Gaussian Sampling in Lattice Based Cryptography. Tatra Mount. Math. Pub. 60, 1 (Sept. 2014), 1-23.

Mark Galassi, Jim Davies, James Theiler, Brian Gough, Gerard Jungman, Patrick Alken, Michael Booth, Fabrice Rossi, and Rhys Ulerich. 2019. GNU Scientific Library. Free Software Foundation.

Paul Glasserman. 2003. Monte Carlo Methods in Financial Engineering. Stochastic Modeling and Applied Probability, Vol. 53. Springer Science+Business Media, New York.

Andrew D. Gordon, Thomas A. Henzinger, Aditya V. Nori, and Sriram K. Rajamani. 2014. Probabilistic Programming. In FOSE 2014: Proceedings of the on Future of Software Engineering (Hyderabad, India). ACM, New York, 167-181.

Te Sun Han and Mamoru Hoshi. 1997. Interval Algorithm for Random Number Generation. IEEE Trans. Inf. Theory 43, 2 (March 1997), 599-611.

Te Sun Han and Sergio Verdú. 1993. Approximation Theory of Output Statistics. IEEE Trans. Inf. Theory 39, 3 (May 1993), 752-772.

John Harling. 1958. Simulation Techniques in Operations Research-A Review. Oper. Res. 6, 3 (June 1958), 307-319.

Eric Jonas. 2014. Stochastic Architectures for Probabilistic Computation. Ph.D. Dissertation. Massachusetts Institute of Technology.

Donald E. Knuth and Andrew C. Yao. 1976. The Complexity of Nonuniform Random Number Generation. In Algorithms and Complexity: New Directions and Recent Results, Joseph F. Traub (Ed.). Academic Press, Inc., Orlando, FL, 357-428.

Dexter Kozen. 2014. Optimal Coin Flipping. In Horizons of the Mind. A Tribute to Prakash Panangaden: Essays Dedicated to Prakash Panangaden on the Occasion of His 60th Birthday. Lecture Notes in Computer Science, Vol. 8464. Springer, Cham, 407-426.

Dexter Kozen and Matvey Soloviev. 2018. Coalgebraic Tools for Randomness-Conserving Protocols. In RAMiCS 2018: Proceedings of the 17th International Conference on Relational and Algebraic Methods in Computer Science (Groningen, The Netherlands). Lecture Notes in Computer Science, Vol. 11194. Springer, Cham, 298-313.

S. Kullback and R. A. Leibler. 1951. On Information and Sufficiency. Ann. Math. Stat. 22, 1 (March 1951), 79-86.

Anthony J. C. Ladd. 2009. A Fast Random Number Generator for Stochastic Simulations. Comput. Phys. Commun. 180, 11 (2009), 2140-2142.

Dopug Lea. 1992. User's Guide to the GNU C++ Library. Free Software Foundation, Inc.

Josef Leydold and Sougata Chaudhuri. 2014. rvgtest: Tools for Analyzing Non-Uniform Pseudo-Random Variate Generators. https://CRAN.R-project.org/package=rvgtest R package version 0.7.4.

Friedrich Liese and Igor Vajda. 2006. On Divergences and Informations in Statistics and Information Theory. IEEE Trans. Inf. Theory 52, 10 (Oct. 2006), 4394-4412.

Jun S. Liu. 2001. Monte Carlo Strategies in Scientific Computing. Springer, New York.

Jérmie Lumbroso. 2013. Optimal Discrete Uniform Generation from Coin Flips, and Applications. (April 2013). arXiv:1304.1916

Vikash Mansinghka and Eric Jonas. 2014. Building Fast Bayesian Computing Machines Out of Intentionally Stochastic Digital Parts. (Feb. 2014). arXiv:1402.4914

The MathWorks. 1993. Statistics Toolbox User's Guide. The MathWorks, Inc.

John F. Monahan. 1985. Accuracy in Random Number Generation. Math. Comput. 45, 172 (Oct. 1985), 559-568.

Aditya V. Nori, Sherjil Ozair, Sriram K. Rajamani, and Deepak Vijaykeerthy. 2015. Efficient Synthesis of Probabilistic Programs In PLDI 2015: Proceedings of the 36th ACM SIGPLAN Conference on Programming Language Design and Implementation (Portland, OR, USA). ACM, New York, 208-217. 
Sung-il Pae and Michael C Loui. 2006. Randomizing Functions: Simulation of a Discrete Probability Distribution Using a Source of Unknown Distribution. IEEE Trans. Inf. Theory 52, 11 (Nov. 2006), 4965-4976.

Karl Pearson. 1900. On the Criterion That a Given System of Deviations from the Probable in the Case of a Correlated System of Variables Is Such That It Can Be Reasonably Supposed to Have Arisen from Random Sampling. Philos. Mag. 5 (July 1900), 157-175.

Yuval Peres. 1992. Iterating von Neumann's Procedure for Extracting Random Bits. Ann. Stat. 20, 1 (March 1992), $590-597$.

R Core Team. 2014. R: A Language and Environment for Statistical Computing. R Foundation for Statistical Computing, Vienna, Austria. http://www.R-project.org/

James R. Roche. 1991. Efficient Generation of Random Variables from Biased Coins. In ISIT 1991: Proceedings of the IEEE International Symposium on Information Theory (Budapest, Hungary). IEEE Press, Piscataway, 169-169.

Sinha S. Roy, Frederik Vercauteren, and Ingrid Verbauwhede. 2013. High Precision Discrete Gaussian Sampling on FPGAs. In SAC 2013: Proceedings of the 20th International Conference on Selected Areas in Cryptography (Burnaby, Canada). Lecture Notes in Computer Science, Vol. 8282. Springer, Berlin, 383-401.

Feras Saad and Vikash Mansinghka. 2016. Probabilistic Data Analysis with Probabilistic Programming. (Aug. 2016). arXiv:1608.05347

Feras A. Saad, Marco F. Cusumano-Towner, Ulrich Schaechtle, Martin C. Rinard, and Vikash K. Mansinghka. 2019. Bayesian Synthesis of Probabilistic Programs for Automatic Data Modeling. Proc. ACM Program. Lang. 3, POPL, Article 37 (Jan. 2019), 32 pages.

Claude E. Shannon. 1948. A Mathematical Theory of Communication. Bell Sys. Tech. Fourn. 27, 3 (July 1948), $379-423$.

Warren D. Smith. 2002. How To Sample from a Probability Distribution. Technical Report DocNumber17. NEC Research.

Sam Staton, Hongseok Yang, Frank Wood, Chris Heunen, and Ohad Kammar. 2016. Semantics for Probabilistic Programming: Higher-order Functions, Continuous Distributions, and Soft Constraints. In LICS 2016: Proceedings of the 31st Annual ACM/IEEE Symposium on Logic in Computer Science (New York, NY, USA). ACM, New York, 525-534.

John Steinberger. 2012. Improved Security Bounds for Key-Alternating Ciphers via Hellinger Distance. Technical Report Report 2012/481. Cryptology ePrint Archive.

Quentin F. Stout and Bette Warren. 1984. Tree Algorithms for Unbiased Coin Tossing with a Biased Coin. Ann. Probab. 12, 1 (Feb. 1984), 212-222.

Tomohiko Uyematsu and Yuan Li. 2003. Two Algorithms for Random Number Generation Implemented by Using Arithmetic of Limited Precision. IEICE Trans. Fund. Elec. Comm. Comp. Sci 86, 10 (Oct. 2003), 2542-2551.

Sridhar Vembu and Sergio Verdú. 1995. Generating Random Bits from an Arbitrary Source: Fundamental Limits. IEEE Trans. Inf. Theory 41, 5 (Sept. 1995), 1322-1332.

John von Neumann. 1951. Various Techniques Used in Connection with Random Digits. In Monte Carlo Method, A. S. Householder, G. E. Forsythe, and H. H. Germond (Eds.). National Bureau of Standards Applied Mathematics Series, Vol. 12. U.S. Government Printing Office, Washington, DC, Chapter 13, 36-38.

Michael D. Vose. 1991. A Linear Algorithm for Generating Random Numbers with a Given Distribution. IEEE Trans. Softw. Eng. 17, 9 (Sept. 1991), 972-975.

Alistair J. Walker. 1974. New Fast Method for Generating Discrete Random Numbers with Arbitrary Frequency Distributions. Electron. Lett. 10, 8 (April 1974), 127-128.

Alastair J. Walker. 1977. An Efficient Method for Generating Discrete Random Variables with General Distributions. ACM Trans. Math. Softw. 3, 3 (Sept. 1977), 253-256. 